\title{
The Hippo effector TAZ cooperates with oncogenic $\beta$-catenin in experimental and human hepatoblastoma development
}

Junyan Tao ( $\sim$ jut24@pitt.edu )

University of Pittsburgh https://orcid.org/0000-0002-5249-970X

Shu Zhang

Sichuan University West China Hospital

Jie Zhang

University of California San Francisco

Katja Evert

University of Regensburgh

Xiaolei Li

PLA 960th Hospital

Pin Liu

Wuhan University Zhongnan Hospital

Andras Kiss

Semmelweis University

\section{Zsuzsa Schaff}

Semmelweis University

Monica Serra

University of Sassari

Matthias Evert

University of Regensburgh

Nianyong Chen

Sichuan University West China Hospital

Feng Xu

Sichuan University West China Hospital

\section{Xin Chen}

University of California, San Francisco

\section{Diego Calvisi}

University of Sassari

Antonio Cigliano

University of Regensburgh 
Research

Keywords: HB, TAZ, $\beta$-catenin, Yap, Notch, HSF1

Posted Date: December 6th, 2019

DOI: https://doi.org/10.21203/rs.2.18319/v1

License: (c) (i) This work is licensed under a Creative Commons Attribution 4.0 International License. Read Full License 


\section{Abstract}

Backgrounds: Hepatoblastoma $(\mathrm{HB})$ is the most common pediatric liver tumor. Though $W n t / \beta$-catenin and Hippo cascades are implicated in HB development, there is no study on the crosstalk of $\beta$-catenin and Hippo downstream effector TAZ in HB.

Methods: The expression of TAZ and of $\beta$-catenin in human HB specimens was assessed by immunohistochemistry (IHC). The functional interplay between TAZ and $\beta$-catenin was tested through delivering either an activated form of TAZ (TAZS89A) alone or co-delivering TAZS89A and an activated form of $\beta$-catenin ( $\triangle N$ N90- $\beta$-catenin) to mouse liver using sleeping beauty transposase via hydrodynamic tail vein injection (SBT-HTVI). In addition, the role of transcriptional enhanced associate domain (TEAD) factors, canonical Notch cascade, Yap, and the tumor modifier heat shock transcription factor 1 (HSF1) along TAZ/ $\beta$-catenin-driven HB development was studied in vivo and vitro.

Results: Activation of TAZ often co-occurred with that of $\beta$-catenin in clinical specimens. While overexpression of TAZS89A alone was unable to promote liver tumorigenesis, the concomitant overexpression of TAZ and $\triangle N 90-\beta$-catenin induced the development of HB lesions exhibiting both epithelial and mesenchymal features. Mechanistically, HB development driven by TAZ/ $\beta$-catenin required TAZ interaction with TEAD factors. Furthermore, TAZ/ $\beta$-catenin overexpression induced HB development in conditional Yes-associated protein knockout (Yap KO) mice, indicating that Yap activation is dispensable in this model. Activation of the Notch signaling was observed in TAZ/ $\beta$-catenin mouse lesions, consistent with that reported in human HBs. Blocking of the canonical Notch cascade using the dominant negative form of RBP-J (dnRBP-J) did not inhibit TAZ/ $\beta$-catenin dependent HB formation in mice, although suppressed the mesenchymal differentiation. Similarly, upregulation of HSF1 was detected in TAZ/ $\beta$-catenin lesions, but its inactivation did not affect HB development. In human HB cell lines, silencing of TAZ resulted in decreased cell growth, which was reduced more substantially when TAZ knockdown was associated with suppression of either $\beta$-catenin or YAP gene.

Conclusions: Overall, our study identifies TAZ as a critical oncogene in HB development and progression. Yap, Notch, and HSF1 are dispensable for TAZ/ $\beta$-catenin induced HB development in mice.

\section{Background}

Hepatoblastoma $(\mathrm{HB})$ is a rare type of cancer, affecting $\sim 1$ to 2 people per million people [1]. It is the most common type of pediatric liver cancer, and mainly occurs in children under 5 years of age. Recently, studies from an international collaboration, Children's Hepatic tumors International Collaboration (CHIC), have been able to stratify the staging of HB based on the clinical information [2]. These studies established the guidelines for selecting the best treatment for HB patients. For primary HB, for instance, surgical resection remains the best and radical therapy for HB. Furthermore, cisplatin-based treatment is the standard chemotherapeutic strategy, and has been used both as adjuvant and neo-adjuvant settings for HB patients [3]. While the long-term survival rate of children with localized HB is excellent, some 
patients are diagnosed with metastatic HB or have relapsed disease. These patients exhibit a poor prognosis, with a 5 -year survival rate of 30 to $40 \%[3,4]$.

HB is considered to originate from primary hepatoblasts and/or hepatic stem cells[4]. Histologically, HB is classified into two major subgroups: epithelial and mixed epithelial/mesenchymal HB. The epithelial subtype can exhibit the phenotype of fetal, embryonal, combined fetal/embryonal or undifferentiated small cells. HBs in the mixed subtype display mesenchymal cells within the tumor lesions [5]. These HB subtype is associated with aggressive growth, invasion, and resistance to chemotherapy [6].

The recent advance of genetics and genomics studies of human HBs provided mechanistic insights into the molecular pathogenesis of this pediatric malignancy. In particular, cumulating evidence supports the activation of the Wnt/ $\beta$-catenin pathway as the driver oncogenic event in HB development. Indeed, oncogenic/activating mutations of CTNNB1 gene, which encodes $\beta$-catenin protein, occur in $60-80 \%$ of human HB samples[4]. Most of the mutations are N-terminal deletions or point mutations in Exon 3 of CTNNB1 gene, leading to the stabilization and nuclear localization $\beta$-catenin. Once in the nucleus, $\beta$ catenin binds to T-cell factor (TCF)/LEF transcription factors, with consequent induction of downstream effectors, including c-MYC, Cyclin D1, and Glutamine Synthase (GS or GLUL), which have been all implicated in tumor development. For instance, in a recent study, it was revealed that glutamine synthetase (GLUL) maintains mTORC1 activation downstream of activated Wnt/ $\beta$-catenin, underlying a novel cell intrinsic regulation of the mTORC1 cascade by the Wnt/ $\beta$-catenin pathway [7]. Besides activating CTNNB1 mutations, inactivating mutations of APC, a major negative regulator of the $\mathrm{Wnt} / \beta$ catenin cascade, also occur in human HB [8], further supporting the pivotal role of the Wnt/ $\beta$-catenin pathway in this disease.

While activation of the Wnt/ $\beta$-catenin cascade has been recognized as a major oncogenic event in $\mathrm{HB}$, this alteration alone has been proven to be insufficient to promote HB formation in vivo. Consequently, it has been suggested that a "second hit" is required to induce HB development in cooperation with activated Wnt/ $\beta$-catenin. In a recent investigation from our group, we found that activation of YAP, a downstream effector of the Hippo tumor suppressor pathway, might represent a second important oncogenic signal in HB [9] .The Hippo signaling is an evolutionary conserved cascade that regulates cell size, metabolism, and growth[10]. In the liver, the Hippo pathway is implicated in modulating liver regeneration, fibrosis, and tumor development [11]. In mammalian cells, Hippo kinases, namely MST1 and MST2, phosphorylate and activate LATS1 and LATS2 kinases. LATS1/2 subsequently phosphorylate YAP and its paralog TAZ. The phosphorylation of YAP and TAZ proteins results in their cytoplasm translocation and degradation[10]. When the Hippo cascade is instead suppressed, YAP and TAZ are unphosphorylated and, therefore, activated. Following activation, YAP and TAZ localize to the nucleus, where they interact with TEAD DNA binding proteins, leading to the increased expression of their target genes, such as CTGF, CYR61, and SURVIVIN [10]. Previously, we have shown the coordinated activation of YAP and Wnt/ $\beta$-catenin in 80\% of human HB samples [9]. In vitro, silencing of YAP inhibited the growth of HB cell lines [9]. Importantly, co-expression of activated forms of YAP (YAPS127A) and $\beta$-catenin $(\Delta \mathrm{N} 90-\beta$-catenin or $\beta$-cateninS45Y) synergizes to promote HB formation in mice (YAPS127A/ $\beta$-catenin) 
[9],[12] .Mechanistically, we discovered that TEAD4 is the major TEAD family member in mouse and human HBs, where it mediates YAPS127A/ $\beta$-catenin driven HB formation [13].

TAZ/WWTR1 (WW Domain Containing Transcription Regulator 1) is a paralog of YAP, and has been found to possess both distinct and redundant roles with YAP in tumorigenesis [14]. While previous studies focused on YAP in HB pathogenesis, the functional role of TAZ in this tumor type has not been defined to date. In this manuscript, we systematically investigated the expression and functions of TAZ in $\mathrm{HB}$ using a comprehensive approach consisting in the analysis of human HB samples, HB cell lines, and mouse models. Our data suggest that TAZ plays an oncogenic role in HB in cooperation with $\beta$-catenin. In this tumor type, TAZ possesses common and distinct functions from YAP.

\section{Materials And Methods}

\section{Human HB samples}

Formalin-fixed, paraffin-embedded tissues from a collection of 32 human HB samples were used for the study. The HB specimens were collected at the Pathology Department of the Semmelweis University (Budapest, Hungary) and the Institute of Pathology at the Medical University of Regensburg (Regensburg, Germany). Clinicopathological features of the human HB samples are reported in Table 1. The study was performed under the Institutional Review Board approval of the local Ethical Committee of the Medical University of Regensburg and the Regional Ethical Committee of the Semmelweis University.

\section{Plasmids and Reagents}

The plasmids used in this study, including pT3-EF1a- $\Delta$ N90- $\beta$-catenin, pT3-EF1a-TAZS89A, pT3-EF1adnRBP-J, pT3-EF1a-dnHSF1, pCMV-Cre, pCMV empty vector, and pCMV/sleeping beauty transposase, were previously described in detail $[9,13]$. The constitutively active form of TAZ (TAZS89A) was purchased from Addgene (plasmid \#24815) and the TEAD-binding deficient form TAZS89AS51A was site mutated using PCR; the two genes were then cloned into the PT3-EF5a plasmid via the Gateway cloning technology (Invitrogen, Carlsbad, CA). All the plasmids for hydrodynamic injection were purified with the Endotoxin-Free Maxiprep kit (Sigma-Aldrich, St. Louis, MO). The pLKO.1 plasmid (empty vector control) was purchased from Addgene (\#10879).

\section{Hydrodynamic Tail Vein Gene Delivery}

FVB/N mice were purchased from Jackson Laboratory (Bar Harbor, ME); and Yap flox/flox mice were provided by Dr. Eric Olson from University of Texas Southwestern Medical Center, Dallas, TX [15]. The hydrodynamic injection procedure has been described in detail in our previous studies [16-19] . Briefly, to

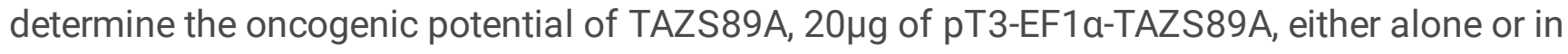


combination with $20 \mu \mathrm{g}$ pT3-EF1 $\mathrm{a}-\Delta \mathrm{N} 90-\beta$-catenin, were mixed together with $\mathrm{pCMV} /$ sleeping beauty transposase at a ratio of 25:1 and injected into 6- to 8-week-old FVB/N mice via the lateral tail vein. To investigate if the oncogenic potential of TAZ was based on TAZ transcriptional function, $20 \mu \mathrm{g}$ of pT3EF1a-TAZS89AS51A were introduced into adult FVB/N mice along with 20 $\mu \mathrm{g}$ pT3-EF1a- $\triangle N$ 90- $\beta$-catenin and $1.6 \mu \mathrm{g} \mathrm{pCMV/sleeping} \mathrm{beauty} \mathrm{transposase.} \mathrm{To} \mathrm{block} \mathrm{the} \mathrm{Notch} \mathrm{cascade,} \mathrm{high} \mathrm{doses} \mathrm{of} \mathrm{dnRBP-J} \mathrm{(60}$ $\mu \mathrm{g})$ together with pT3-EF1a-TAZS89A $(20 \mu \mathrm{g})$ and pT3-EF1a- $\triangle$ N90- $\beta$-catenin $(20 \mu \mathrm{g})$ plasmids were injected. In addition, to assess the importance of HSF1 in TAZ/ $\beta$-catenin dependent HB formation, we injected $20 \mu \mathrm{g}$ of pT3-EF1a-TAZS89A and $20 \mu \mathrm{g}$ of pT3-EF1a- $\triangle N$ N0- $\beta$-catenin together with $60 \mu \mathrm{g}$ of either pT3-EF1a-dnHSF1 or pCMV empty vector. Finally, to study if YAP is required for TAZ-dependent HB

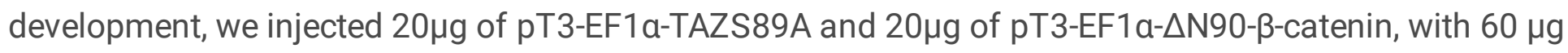
of either $\mathrm{pCMV}$-Cre or pCMV empty vector, as well as pCMV/sleeping beauty transposase into adult Yap flox/flox mice. All mice used in the experiments were monitored continually and euthanized at specific time points, as indicated in the main text, or when they became moribund. Mice were maintained and monitored in accordance with protocols approved by the Committee for Animal Research at the University of California, San Francisco (San Francisco, CA).

\section{Histology, Immunohistochemistry, Assessment of Proliferation, Apoptosis, and Microvessel Density}

Liver specimens were harvested and fixed in $10 \%$ formalin overnight at $4^{\circ} \mathrm{C}$ and embedded in paraffin. Hematoxylin and eosin (ThermoFisher Scientific, Waltham, MA) staining was conducted using a standard protocol on human and mouse liver sections and slides analyzed by two expert liver pathologists (KE and $\mathrm{ME})$ in accordance with the criteria described before[9, 20]. Immunohistochemistry (IHC) was performed according to our previous studies[9, 13]. Proliferation index was determined in mouse HB lesions by counting Ki-67 positive cells on at least 3000 tumor cells per mouse sample. Apoptosis index was determined in mouse HCC lesions by counting TUNEL positive cells on at least 3000 tumor cells per mouse using the TumorTACS ${ }^{\mathrm{TM}}$ In Situ Apoptosis Detection Kit (Trevigen, Gaithersburg, MD), following the manufacturer's instructions. Assessment of microvessel density (MVD) was conducted as previously reported [21]. Specifically, liver tumor samples from YAPS127A/ $\Delta N 90-\beta$-catenin and TAZS89A/ $\Delta N 90-\beta-$ catenin mice were subjected to immunostaining with the mouse monoclonal anti-CD34 antibody (Abcam, United Kingdom). The tumors were first screened at low power $(\times 40)$ to identify the areas of highest microvessel density (MVD). The four highest MVD areas for each tumor were photographed at high power $(\times 200)$ and the size of each area standardized using the ImageJ software. Any brown-stained endothelial cell or endothelial cell cluster was counted as one microvessel, irrespective of the presence of a vessel lumen. MVD was expressed as the percentage (mean $\pm S D)$ of the total CD34-stained spots per section area $\left(0.94 \mathrm{~mm}^{2}\right)$. The primary antibodies used in the study are described in Table 2.

\section{Western Blot Analysis}


Frozen liver tissues and cell pellets were homogenized in Mammalian Protein Extraction Reagent (ThermoFisher Scientific) containing the phosphatase inhibitors. Protein concentration was determined using the Bio-Rad Protein Assay kit (Bio-Rad, Hercules, CA) using bovine serum albumin as a standard. Supernatants were denatured by boiling in Tris-Glycine SDS Sample Buffer (Life Technologies, Carlsbad, CA) for Western blot analysis. Proteins were separated by SDS PAGE and transferred onto nitrocellulose membranes (Life Technologies) by electroblotting. Membranes were blocked in 5\% non-fat dry milk for 1 $\mathrm{h}$ and then incubated with proper primary antibodies (Table 2). Subsequently, membranes were incubated with horseradish peroxidase-secondary antibodies (Jackson Immunoresearch Laboratories Inc., West Grove, PA, USA) diluted 1:5000 for 30 min. Proteins bands were revealed with the Super Signal West Femto (Pierce Chemical Co., New York, NY).

\section{RNA extraction and quantitative real-time reverse transcriptase polymerase chain reaction (qRT-PCR)}

Total mRNA from liver tissues and cells was extracted by using the Quick RNA Miniprep kit (Zymo Research, Irvine, CA, USA). Next, mRNA expression of the genes of interest was detected by quantitative real-time polymerase chain reaction (qRT-PCR) using validated Gene Expression Assays for human and mouse genes (ThermoFisher Scientific; Table 3). PCR reactions were performed with $100 \mathrm{ng}$ of cDNA of the collected samples or cell lines, using an ABI Prism 7000 Sequence Detection System with TaqMan Universal PCR Master Mix (Applied Biosystems). Cycling conditions were as follows: denaturation at $95^{\circ} \mathrm{C}$ for $10 \mathrm{~min}, 40$ cycles at $95^{\circ} \mathrm{C}$ for $15 \mathrm{~s}$, and then extension at $60^{\circ} \mathrm{C}$ for $1 \mathrm{~min}$. Quantitative values were calculated by using the PE Biosystems Analysis software and expressed as $N$ target (NT). NT $=2^{-\Delta C t}$, wherein the $\Delta \mathrm{Ct}$ value of each sample was calculated by subtracting the average $\mathrm{Ct}$ value of the target gene from the average $\mathrm{Ct}$ value of the $\beta$-Actin gene.

\section{Cell lines and In vitro study}

The Hep293TT cell line was a kind gift of Dr. Gail Tomlinson (University of Texas Southwestern Medical Center, Dallas, TX). The HepG2 cell line was purchased from ATCC (Manassas, VA). Both cell lines were authenticated (Genetica DNA Laboratories, Burlington, NC) and tested clear of mycoplasma contamination. They were maintained as described in our previous study and cultured at $37^{\circ} \mathrm{C}$ in $5 \% \mathrm{CO} 2$ humidified incubator. For knockdown studies in vitro, HepG2 and Hep293TT cell lines were transfected with small interfering RNA (siRNA) against human TAZ (\# s24789), $\beta$-catenin (\# s436), and/or YAP (\# s20366) or scrambled siRNA (\# s4390846, negative control; ThermoFisher Scientific) via using Lipofectamine $^{\text {TM }}$ RNAiMAX Transfection Reagent (ThermoFisher Scientific) according to the manufacturer's instructions. Cell proliferation was assessed in HepG2 and Hep293TT cell lines at the 48hour time point using the BrdU Cell Proliferation Assay Kit (Cell Signaling Technology, Danvers, MA, USA). As concerns apoptosis, it was determined in the same cell lines using the Cell Death Detection Elisa plus 
Kit (Roche Molecular Biochemicals, Indianapolis, IN, USA), following the manufacturer' instructions. All experiments were repeated at least three times in triplicate.

\section{Statistical Analysis}

All data are presented as mean \pm SD for each group. Statistical differences between two groups were determined using the U-tests embedded in the Prism 6 software version 6.0 (Graph Pad Software Inc., La Jolla, CA). $P<0.05$ was considered statistically significant.

\section{Results}

\section{Frequent activation of TAZ in human HB samples}

First, we assessed the levels of TAZ protein in a collection of human HB specimens $(n=32)$ by IHC (Figure 1). In non-tumorous surrounding liver, cytoplasmic and nuclear positive staining for TAZ was limited to biliary cells, whereas hepatocytes showed either absent or faint cytoplasmic immunolabeling for this protein, in accordance with previous data [22]. An equivalent staining pattern was detected for the TAZ paralog, YAP, as well as for NOTCH2, a TAZ target. As expected, $\beta$-catenin protein was expressed in the membrane of hepatocytes as well as in the membrane and cytoplasm of biliary cells. Noticeably, strong cytoplasmic and nuclear immunoreactivity for TAZ was detected in most HB specimens (22/32; $68.7 \%)$. The frequency was lower than that found for nuclear $\beta$-catenin $(27 / 32,84.4 \%)$ and the TAZ paralog YAP $(26 / 32,87.5 \%)$ but higher than nuclear NOTCH2 (19/32; 59.4\%) in the same HB collection. Concomitant nuclear immunoreactivity for TAZ and $\beta$-catenin was detected in 18 of 22 TAZ-positive tumors $(81.8 \%)$, while 15 of $22(71.4 \%)$ human HB with TAZ activation displayed positive nuclear immunolabeling for YAP and NOTCH2. No significant association was found between the levels of TAZ, $\beta$-catenin, YAP, and NOTCH2 with clinicopathologic features of the patients, including age, gender, histology subtype, recurrence, or lung metastasis (data not shown).

Overall, the present data unravel the frequent activation of TAZ in human $\mathrm{HB}$, which is frequently associated with induction/overexpression of YAP, $\beta$-catenin, and NOTCH2.

\section{Constitutively activated TAZ (TAZS89A) cooperates with oncogenic $\beta$-catenin to promote HB development in mice}

To elucidate the functional role of TAZ along HB pathogenesis, we investigated whether activated TAZ can either induce liver carcinogenesis or cooperate with other oncogenes to promote HB formation in mice. For this purpose, we hydrodynamically transfected the constitutively activated form of TAZ 
(TAZS89A) and the $N$-terminal deleted activated mutant form of $\beta$-catenin ( $\triangle N$ N90- $\beta$-Catenin), alone or in combination, in the mouse liver (Figure 2A). TAZS89A is a mutant form allowing TAZ to escape phosphorylation-mediated degradation and, thus, leading to TAZ unrestrained activity [23].

Overexpression of TAZS89A alone did not lead to liver tumor formation in mice. Indeed, 21 weeks post hydrodynamic injection, all mice appeared to be healthy. Accordingly, upon dissection, no tumor nodule was detected. Subsequent histological evaluation revealed that the mouse liver was completely normal, lacking any histological alteration (Figure $2 \mathrm{~B}, \mathrm{C}$ ). As previous studies showed that overexpression of $\triangle \mathrm{N} 90-\beta$-catenin alone does not lead to liver tumor development in mice but cooperates with other oncogenes for cancer formation [24-26], this $\beta$-catenin mutant form was co-injected with TAZS89A in the mouse liver (TAZS89A/ $\triangle N$ N90- $\beta$-catenin mice) (Figure 2A). Noticeably, concomitant overexpression of TAZS89A with $\Delta$ N90- $\beta$-catenin resulted in rapid liver tumor formation in TAZS89A/ $\Delta N 90-\beta$-catenin mice. By 6 weeks post injection, $100 \%$ of the injected mice developed high liver tumor burden with palpable abdominal mass and were euthanized based on our IACUC protocol (Figure 2B,D,E).

Microscopically, small cluster of cells could be visualized as early as $\sim 1.5$ weeks post injection in the liver of TAZS89A/ $\Delta$ N90- $\beta$-catenin mice (Figure 3A). Positive immunoreactivity for Myc Tagged- $\Delta$ N90- $\beta$ catenin and TAZ antibodies confirmed that tumor cells originated from the injected plasmids (Figure $3 B, C)$, while immunolabeling for Ki67 implied active cell proliferation (Figure 3D). The first tumor nodules were visible in TAZS89A/ $\Delta$ N90- $\beta$-catenin mice by 3 weeks post injection (Figure $3 E$ ). Tumors quickly occupied nearly completely the liver parenchyma by 6 weeks post injection (Figure 3F).

Histologically, most of the lesions consisted of small cells with prominent nuclei, similar to those described in YAPS127A/ $\beta$-catenin mice $[9,12]$, and resembling human fetal or crowded fetal HB (Figure $3 \mathrm{G}, \mathrm{H})$. Nonetheless, a second, distinct tumor entity was appreciable in the liver parenchyma of TAZS89A/ $\Delta$ N90- $\beta$-catenin mice. Indeed, besides the "pure" hepatoblast-like lesions, many tumors displayed a mixed phenotype, consisting of an epithelial (hepatoblast) and a mesenchymal component, with the latter being characterized primarily by cells with spindle morphology (Figure $3 \mathrm{~J}, \mathrm{~K}$ ). To the best of our knowledge, this is the first reported mouse model showing features of epithelial and mesenchymal differentiation. Following this interesting observation, we sought to determine the molecular characteristics of the two tumor entities by using IHC (Figure 4). As expected, both types of lesions displayed immunoreactivity for Myc Tagged- $\Delta$ N90- $\beta$-catenin and TAZ antibodies, implying their origin from the injected constructs. Increased levels of the injected constructs were confirmed by Western blot analysis (Supplementary Figure 1). In addition, immunoreactivity for hepatocellular markers (hepatocyte nuclear factor 4 alpha or HNF-4a, forkhead box A1/A2 or FOXA1/A2, CCAAT Enhancer Binding Protein Alpha or CEBPA, and glutamine synthetase or GLUL) was significantly more pronounced in hepatoblast-like lesions. Furthermore, levels of the progenitor markers CD44v6, NANOG, CD10, and EPCAM as well as the cholangioblastic markers cytokeratin 7 and 19 (CK7 and CK19) were higher in the epithelial lesions. 
Levels of the GATA binding factor 4 (GATA4), whose expression is frequently upregulated in human HB with mesenchymal features[27], were strongly induced in both tumor entities. The mesenchymal part of the lesions, on the other hand, showed higher levels of stromal and angiogenesis-related markers, such as ERG, CD34, alpha-smooth muscle actin (a-SMA), and CD56/NCAM1, whereas immunoreactivity for the epithelial marker E-Cadherin ( $\mathrm{CDH} 1)$ was lost in the same mesenchymal lesions (Supplementary Figure 2).

Furthermore, by real-time RT-PCR, we found that livers of TAZS89A/ $\triangle$ N90- $\beta$-catenin mice exhibited significantly higher levels than wild-type livers of glypican 3 (Gpc3), alpha-fetoprotein (Afp), Cited1, DIk1, and Gata4 genes (Supplementary Figure 3), whose upregulation in human HB is well established [27, 28] . As expected, mRNA expression of $\beta$-catenin (glutamine synthetase or G/ul, Axin2, and TBX3) and TAZ (connective tissue growth factor or CTGF, Cysteine-Rich Heparin-Binding Protein 61 or Cyr61, Jagged 1, Notch1, Notch2, and Hes1) canonical targets was more elevated in TAZS89A/DN90- $\beta$-catenin livers when compared with the wild-type counterpart (Supplementary Figure 4).

\section{TAZS89A $\triangle N$ N0- $\beta$-catenin induced hepatoblastoma requires an intact TAZ interaction with TEAD transcription factors}

TAZ is a transcriptional activator and it interacts with TEAD DNA binding proteins to induce downstream gene expression. Nonetheless, TAZ is also known to have functions independent of its binding to TEAD factors $[10,11,29]$. Thus, we sought to investigate whether TAZS89A/ $\triangle N 90-\beta$-catenin induced HB development requires an intact TAZ/TEAD interaction. To address this issue, we generated the TAZS89AS51A plasmid for hydrodynamic transfection. The S51A mutation prevents TAZ from binding to TEAD proteins [29]. Therefore, we co-expressed TAZS89AS51A and $\triangle N 90-\beta$-catenin plasmids into the mouse liver (Figure 5A). All TAZS89AS51A/ $\Delta$ N90- $\beta$-catenin mice appeared to be healthy and were harvested at 5-8 weeks post injection (Figure 5B). Grossly, no tumors nodules were detected on the mouse liver. Subsequent histological analysis revealed that the liver tissues of TAZS89AS51A/ $\Delta N 90-\beta$-catenin mice was completely normal, and the liver weight/body weight ratio of these mice was equivalent of that of wild-type, un-injected liver (Figure 5C,D). Altogether, the present data demonstrate that TEAD proteins are required for TAZ driven HB development in mice.

\section{Commonalities and differences between TAZS89A $\Delta$ N90- $\beta$-catenin and YAPS127A $\Delta$ N90- $\beta$-catenin lesions}

Previously, we reported that the activated form of Yap (YAPS127A), the other oncogenic transducer of the Hippo signaling, cooperates with $\triangle N 90-\beta$-catenin to induce HB formation in vivo [9]. Thus, we compared the liver tumors developed in TAZS89A/ $\Delta N 90-\beta$-catenin and YAPS127A/ $\Delta N 90-\beta$-catenin mice. 
Histologically, "pure" hepatoblast-like lesions were undistinguishable between the two models. However, mesenchymal lesions were absent in YAPS127A/ $\Delta$ N90- $\beta$-catenin mice (Supplementary Figure $5 A$ ). At the cellular level, the rates of proliferation, apoptosis, and angiogenesis (microvessel density; MVD) were compared between the epithelial lesions from TAZS89A/ $\Delta$ N90- $\beta$-catenin and YAPS127A/ $\Delta$ N90- $\beta$-catenin mice. No significant differences were observed in the rates of proliferation and MVD between the two mouse models, whereas apoptosis was significantly lower in TAZS89A/ $\Delta$ N90- $\beta$-catenin mice (Supplementary Figure 5B-D).

At the molecular level, TAZS89A/ $\Delta$ N90- $\beta$-catenin and YAPS127A/ $\Delta$ N90- $\beta$-catenin tumors expressed $A f p$ and Gpc3 genes at similar levels. In addition, YAPS127A/ $\triangle$ N90- $\beta$-catenin mouse malignant lesions expressed EpCam at higher levels, whereas TAZS89A/ $\triangle$ N90- $\beta$-catenin tumors displayed higher levels of HB markers DIk1 and Cited 1 as well as of the Glul hepatocellular marker. TAZS89A/ $\Delta$ N90- $\beta$-catenin tumors also exhibited the highest mRNA expression of the Gata4 transcription factor (Supplementary Figure 6). By immunohistochemistry, we found that levels of hepatocellular markers HNF-4a, FOXA2, FOXA1, CEBPA (the last two not shown), were equivalent in the two models. In contrast, GLUL protein was expressed homogeneously in TAZS89A/ $\triangle$ N90- $\beta$-catenin malignant lesions, whereas only patchy immunoreactivity for this protein was detected in YAPS127A/ $\triangle N 90-\beta$-catenin corresponding lesions. No differences were detected in the levels of the progenitor markers CD44v6, and CD10 as well as the cholangioblast markers CK7 and CK19 in the two models, whereas staining intensity for EPCAM was significantly stronger and more homogeneous in YAPS127A/ $\triangle$ N90- $\beta$-catenin tumors (Supplementary Figure 7), in accordance with real-time RT-PCR data.

Finally, we determined the levels of two transcription factors, whose role in liver cancer is becoming more and more relevant, namely nuclear factor erythroid 2 like 2 (NFE2L2/NRF2) and heat shock factor transcription factor 1 (HSF1) in TAZS89A/ $\triangle$ N90- $\beta$-catenin and YAPS127A/ $\Delta$ N90- $\beta$-catenin tumors[3032]. Levels of NRF2, its regulator Kelch like ECH associated protein 1 (Keap1), and those of its target $\mathrm{NAD}(\mathrm{P}) \mathrm{H}$ quinone dehydrogenase 1 (Nqo1), were upregulated in both mouse models, without statistical differences between the two cohorts, when compared with wild-type mice (Supplementary Figure 8). In striking contrast, a robust overexpression of HSF1 was observed only in TAZS89A/ $\Delta N 90-\beta$-catenin mouse lesions both via RT-PCR and IHC (Supplementary Figure 8). Altogether, the present data indicate the existence of common and distinct features between TAZS89A/ $\triangle N$ N0- $\beta$-catenin and YAPS127A/ $\Delta \mathrm{N} 90-\beta$-catenin tumors.

HSF1 is dispensable along TAZS89A $\Delta N 90-\beta$-catenin dependent hepatoblastoma development 
Following the interesting finding of upregulation of HSF1 exclusively in TAZS89A/ $\Delta$ N90- $\beta$-catenin tumors, we assessed the levels of HSF1 in human HB specimens by IHC to determine a possible role of HSF1 in this tumor type. Of note, HSF1 was ubiquitously $(32 / 32,100 \%)$ upregulated and localized in the nucleus of HB cells in the collection examined (Supplementary Figure 9), whereas low/absent immunoreactivity for HSF1 was detected in corresponding surrounding non-tumorous livers. Subsequently, the effect of HSF1 inactivation was investigated in the TAZS89A/ $\Delta$ N90- $\beta$-catenin mouse model. Specifically, to achieve this goal, we co-injected TAZS89A and $\Delta$ N90- $\beta$-catenin plasmids with a dominant negative form of HSF1 (dnHSF1) in the mouse liver via hydrodynamic gene delivery (these mice will be referred to as TAZS89A/ $\triangle N$ 90- $\beta$-catenin/dnHSF1) (Figure 6A). This approach was found to be highly effective in completely suppressing liver carcinogenesis driven by either AKT or c-Myc protooncogene in mice $[33,34]$. Unexpectedly, HSF1 inactivation neither affected HB development (Figure $6 \mathrm{~B})$ nor changed the histopathological features of TAZS89A/ $\triangle N$ N90- $\beta$-catenin mice (Figure $6 \mathrm{C}$ ). At the microscopical level, in fact, both epithelial and mesenchymal features were retained by HB lesions developed in TAZS89A/ $\triangle$ N90- $\beta$-catenin/dnHSF1 mice (Figure 6C). Furthermore, no significant differences were detected in liver/body weight ratio between TAZS89A/ $\triangle$ N90- $\beta$-catenin and TAZS89A/ $\Delta$ N90- $\beta$ catenin/dnHSF1 mice (Figure 6D).

Taken together, the present data indicate that TAZS89A/ $\triangle$ N90- $\beta$-catenin HB development does not primarily depend on HSF1 in mice.

\section{Loss of endogenous YAP does not affect TAZS89A $\Delta$ N90- $\beta$-catenin driven hepatoblastoma formation}

Next, we asked whether activated YAP and TAZ have redundant and/or distinct roles in HB formation. First, we evaluated the levels of YAP, the TAZ paralog, in HB lesions from TAZS89A/ $\triangle N 90-\beta$-catenin mice. We found that, while total protein levels of YAP in TAZS89A/ $\triangle$ N90- $\beta$-catenin lesions were equivalent to those in wild-type livers (Supplementary Figure 1), nuclear accumulation of YAP (a sign of its activation) was detected in TAZS89A/ $\triangle$ N90- $\beta$-catenin HB lesions (Figure 7). Subsequently, we investigated whether TAZS89A/ $\triangle$ N90- $\beta$-catenin overexpression was able to promote HB development in mice in the absence of endogenous YAP protein. For this purpose, we applied conditional Yap KO (Yap flox/loxf mice[15]. Specifically, we hydrodynamically injected TAZS89A/ $\triangle N$ N0- $\beta$-catenin plasmids together with pCMV-Cre plasmids into Yap flox/flox mice (TAZS89A/ $\Delta$ N90- $\beta$-catenin/Cre). As the control, TAZS89A/ $\triangle N 90-\beta$-catenin plasmids were mixed with pCMV empty vector and co-injected into Yap flox/flox mice (TAZS89A/ $\Delta$ N90- $\beta$ catenin/pCMV) (Figure 7A). We found that both cohorts of mice developed liver tumors and were required to be euthanized by 10 to 12 weeks post injection (Fig. 7B and 7D). Histologically, HB lesions were detected in TAZS89A/ $\Delta$ N90- $\beta$-catenin/pCMV and TAZS89A/ $\triangle$ N90- $\beta$-catenin/Cre injected mice, consisting of both the epithelial and the mesenchymal component (Fig. 7D). Importantly, while cytoplasmic and nuclear staining of YAP could be detected in TAZS89A/ $\triangle N 90-\beta$-catenin/pCMV HB cells, 
no YAP expression was found in TAZS89A/ $\Delta$ N90- $\beta$-catenin/Cre HB cells. Instead, positive YAP staining was readily observed in the surrounding normal liver tissues as well as stromal cells within the HB lesions (Fig. 7D). Loss of YAP expression in TAZS89A/ $\Delta$ N90- $\beta$-catenin/Cre tumor tissues was further validated by Western blotting (Fig. 7C).

Overall, the present data indicate that activated TAZ induces HB formation in the absence of the endogenous YAP.

\section{Canonical Notch signaling is not required for TAZS89A $\Delta$ N90- $\beta$-catenin HB development in vivo}

Mounting evidence indicate the Notch pathway as a downstream effector of the Hippo cascade in liver cancer [35-37]. In addition, NOTCH2 has been found to be consistently overexpressed in human HB [38].

Furthermore, our present data indicate the upregulation of members of the Notch pathway (Jag1, Notch1, Notch2, and Hes1) in the lesions of TAZS89A/ $\triangle$ N90- $\beta$-catenin mice. Thus, we investigated whether the canonical Notch signaling is required for TAZS89A/ $\triangle$ N90- $\beta$-catenin driven HB formation in vivo. For this purpose, we hydrodynamically injected TAZS89A/ $\triangle$ N90- $\beta$-catenin plasmids together with the dominant negative form of RBP-J (dnRBP-J; these mice will be referred to as TAZS89A/ $\Delta$ N90- $\beta$-catenin/dnRBP-J) that has been shown to effectively inhibit the canonical Notch cascade (Figure 8A) [37, 39]. Additional mice were injected with TAZS89A/ $\Delta$ N90- $\beta$-catenin with $\mathrm{pT} 3$ empty vector as control (TAZS89A/ $\Delta N 90-\beta$ catenin/pT3). We found that all mice develop high liver tumor burden and were required to be euthanized around 6 to 9 weeks post injection (Figure 8B-D). Histologically, HB lesions could be appreciated in both cohorts of mice. However, overexpression of dnRBP-J in the livers of TAZS89A/ $\Delta$ N90-B-catenin mice led to the disappearance of the mesenchymal compartment of HB lesions (Figure $8 \mathrm{C}$ ), indicating that the Notch pathway might contribute to the differentiation toward a mesenchymal phenotype of TAZS89A/ $\Delta$ N90- $\beta$-catenin HB lesions.

Overall, our study indicates that Notch signaling is activated in TAZS89A/ $\triangle N 90-\beta$-catenin tissues and influences the tumor cell differentiation. However, Notch activation is dispensable in TAZS89A/ $\triangle N 90-\beta-$ catenin driven HB development.

\section{TAZ cooperates with $\beta$-catenin and YAP for the growth of human HB cells in vitro}

The findings in human and mouse HB specimens support a major role of TAZ in HB formation. To further investigate the relevance of TAZ activation in this tumor type, we examined whether TAZ expression is required for human HB cell growth in vitro. Thus, we silenced TAZ in two human HB cell lines (HepG2 and 
Hep293TT cells) using specific siRNA against TAZ (Supplementary Figure 10, 11). Of note, TAZ knockdown resulted in a decrease of cell proliferation and induction of apoptosis in the two HB cell lines. These anti-growth effects were increased when the two cell lines were concomitantly subjected to TAZ and $\beta$-catenin silencing. At the molecular level, suppression of TAZ did not affect $\beta$-catenin and YAP mRNA and protein levels, whereas $\beta$-catenin knockdown resulted in downregulation of TAZ and YAP.

Finally, we assessed whether TAZ and YAP have redundant roles in human HB cells. For this purpose, we silenced TAZ and YAP genes using specific siRNAs, either alone or in combination, in HepG2 and Hep293TT cell lines (Supplementary Figure 12,13). We found that concomitant knockdown of TAZ and YAP resulted in a significantly more pronounced growth restraint when compared with the silencing of either TAZ or YAP alone. At the molecular level, knockdown of TAZ did not affect the levels of YAP, whereas silencing of YAP triggered upregulation of TAZ. These findings suggest the existence of a compensatory mechanism triggering upregulation of TAZ when YAP is suppressed in HB cells.

Altogether, the present data indicate that TAZ acts in concert with $\beta$-catenin and YAP to induce HB growth in vitro.

\section{Discussion}

$\mathrm{HB}$ is the most frequent pediatric tumor of the liver [1]. The cellular and molecular mechanisms underlying HB development and progression remain poorly delineated. Recently, a body of evidence has unraveled the crucial role in this disease of the Wnt/ $\beta$-catenin signaling, which is aberrantly activated virtually in all cases $[4,7,8]$. Nonetheless, overexpression of oncogenic forms of $\beta$-catenin does not suffice to induce HB formation in mice, implying the need of additional alterations to drive HB development. Recently, we showed that $\beta$-catenin cooperates with the YAP protooncogene, a downstream effector of the Hippo pathway, to induce rapid HB formation in the mouse liver [9]. These data, together with the findings of simultaneous activation of $\beta$-catenin and YAP in human HB specimens [9], strongly support the functional importance of the Hippo pathway in this disease. In the present study, we further investigated the role of the Hippo signaling in this tumor type by focusing on the YAP ortholog, TAZ. First, we showed the frequent activation of TAZ in human HB specimens, suggesting an important role of TAZ in this tumor type. Furthermore, we demonstrated that the activation of TAZ synergizes with oncogenic $\beta$ catenin to induce HB formation in mice, thus recapitulating the effects of combining YAP and $\beta$-catenin overexpression in the mouse liver. Nonetheless, some important differences occur between TAZS89A/ $\triangle N$ 90- $\beta$-catenin and YAPS127A/ $\triangle N$ 90- $\beta$-catenin HB lesions. In particular, histopathological analysis revealed that HB lesions from TAZS89A/ $\triangle N 90-\beta$-catenin display the presence of a mesenchymal component, which is instead absent in corresponding lesions from YAPS127A/ $\Delta$ N90- $\beta$-catenin mice. Although the molecular reason(s) for this histopathologic difference between the two HB models is not clear, our data suggests that the Notch pathway plays a major role in this phenomenon. Indeed, suppression of the canonical Notch cascade via the use of a dnRBP-J was accompanied by the 
disappearance of the mesenchymal phenotype in TAZS89A/ $\Delta$ N90- $\beta$-catenin HB lesions. Additional studies are needed to better define the molecular mechanisms and the specific Notch targets involved in this event.

Another gene specifically upregulated in TAZS89A/ $\triangle N 90-\beta$-catenin tumors was the HSF1 transcription factor, whose oncogenic role in liver cancer is well-established [15, 32, 33]. Unexpectedly, inactivation of HSF1 in TAZS89A/ $\triangle$ N90- $\beta$-catenin mice had no effect on HB development. Previously, we showed that blockade of HSF1 activity using the same strategy is able to completely suppress hepatocarcinogenesis driven by either AKT or c-Myc overexpression in mice[15,33]. These contrasting data suggest that the addiction to HSF1 is presumably oncogene- and context dependent in cancer. Similarly, by conducting

loss of function experiments using Yap ${ }^{\text {flox/flox }}$ conditional knockout mice, we discovered that inhibition of YAP neither suppresses nor delays HB development in TAZS89A/ $\Delta$ N90- $\beta$-catenin mice. These in vivo findings, together with in vitro data showing an additive anti-growth effect of simultaneous silencing of TAZ and YAP in human HepG2 and Hep293TT HB cell lines, indicate that the functions of TAZ and YAP might only partly overlap in this tumor entity, at least in mice. In addition, preliminary results from our group indicate that concomitant overexpression of YAPS127A and TAZS89A in the mouse liver does not suffice to drive tumor development (Calvisi et al., unpublished observation). The latter observation suggests that YAP and TAZ are not able to trigger liver tumor initiation alone or in combination, at least by the hydrodynamic gene delivery method. However, these findings do not exclude a collaborative (and non-redundant) role of the two protooncogenes in driving tumor progression. Further studies using TAZ and YAP conditional knockout mice should be conducted to address this important issue.

Our present data might also have potentially important therapeutic implications for this aggressive disease in humans. Indeed, the findings from mouse models and cell lines suggest that concomitant targeting of the Wnt/ $\beta$-catenin and Hippo pathways using specific inhibitors might be highly detrimental for HB growth. Unfortunately, these two signaling cascades are not easily druggable and no effective drugs against them are commercially available. A possible strategy would be the use of Tankyrase inhibitors, which are able to suppress both oncogenic pathways and recently provided encouraging results for the treatment of hepatocellular carcinoma $[40,41]$.

\section{Conclusions}

In summary, our findings for the first time support a critical oncogenic function of TAZ in the development of $\mathrm{HB}$ as well as its collaborative role with the $\beta$-catenin signaling pathway in this aggressive disease. Further studies using the TAZS89A/ $\triangle N$ N90- $\beta$-catenin mouse model would be necessary to elucidate the mechanisms of such cooperation and might provide a deeper understanding of the molecular mechanisms underlying the pathogenesis of human and its treatment.

\section{Abbreviations}


HB: hepatoblastoma; TEAD: transcriptional enhanced associate domain; HSF1: heat shock transcription factor 1; dominant negative form of HSF1 (dnHSF1); IHC: immunohistochemistry; SBT-HTVI区 sleeping

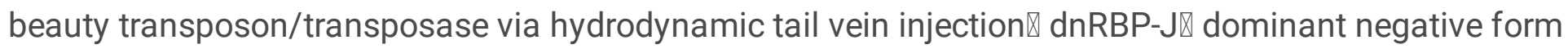
of RBP-J『CHIC区 Children's Hepatic tumors International Collaboration囚TAZ/WWTR1 WW Domain Containing Transcription Regulator 1; MVD: microvessel density; Keap1: Kelch like ECH associated protein; Nqo1: NAD $(P) H$ quinone dehydrogenase 1.

\section{Declarations}

\section{Ethics approval and consent to participate}

Ethical approval was performed under the Institutional Review Board approval of the local Ethical Committee of the Medical University of Regensburg and the Regional Ethical Committee of the Semmelweis University, and written informed consent was obtained from each patient.

\section{Consent for publication}

Not applicable.

\section{Availability of supporting data}

All data developed or analyzed during this study are presented either in this article or in the Supplementary Materials and Methods, Tables, Figures and Figure Legends files.

\section{Competing interests}

The authors declare that they have no competing interests.

\section{Funding}

Diego F. Calvisi was supported by a grant from the Italian Association Against Cancer (AIRC), grant number IG19175; Xin Chen was supported by a NIH grant (R01CA204586). This work was also supported by multiple grants from National Natural Science Foundation of China: 81573024 (Feng Xu), 81802774 (Pin Liu), 81702448 (Xiaolei Li). 


\section{Authors' Contributions:}

SZ, technical and material support; analysis and interpretation of data; JZ, technical and material support and analysis and interpretation of data; KE, Pathology support; XLL, technical and material support; PL, technical and material support; AK, technical support; ZS, technical and material support and important intellectual content; MS, technical and material support; ME, Pathology support; critical revision of the manuscript; NYC, study design; FX, analysis and interpretation of data; $X C$, study design, drafting and critical revision of manuscript; JYT, technical and material support, revision of manuscript; DFC, study design, drafting and critical revision of manuscript; AC, study design, technical support; critical revision of manuscript. All authors read and approved the final manuscript.

\section{Acknowledgements}

We thank Ms. Ingrid Winkel and Mr. Manfred Meyer (Institute of Pathology, University of Regensburg, Regensburg, Germany) for their excellent technical work; Dr. Gail Tomlinson from University of Texas Southwestern Medical Center for providing us with Hep293TT cells; as well as Dr. Eric Olson from the University of Texas Southwestern Medical Center for providing Yap $p^{\text {flox/flox }}$ mice.

\section{Authors' information}

${ }^{1}$ Cancer Center, West China Hospital, Sichuan University, Chengdu, Sichuan, P.R.China; ${ }^{2}$ Department of Bioengineering and Therapeutic Sciences and Liver Center, University of California, San Francisco, San Francisco, California, USA; ${ }^{3}$ Department of Thoracic Oncology II, Key Laboratory of Carcinogenesis and Translational Research (Ministry of Education), Peking University Cancer Hospital and Institute, Beijing, P.R.China; ${ }^{4}$ Institute of Pathology, University of Regensburg, Regensburg, Germany; ${ }^{5}$ Department of Thyroid and Breast Surgery, the $960^{\text {th }}$ Hospital of the PLA, Jinan, Shandong, P.R. China; ${ }^{6}$ Department of Pediatrics, Zhongnan Hospital of Wuhan University, Wuhan, Hubei, P.R.China; ${ }^{7}$ Second Department of Pathology, Semmelweis University, Budapest, Hungary; ${ }^{8}$ Department of Medical, Surgical, and Experimental Sciences, University of Sassari, Sassari, Italy; ${ }^{9}$ Lung Cancer Center, West China Hospital, Sichuan University, Chengdu, Sichuan, P.R.China; ${ }^{10}$ Department of Pathology, University of Pittsburgh School of Medicine, Pittsburgh, Pennsylvania, USA.

\section{References}

1. Czauderna P, Haeberle B, Hiyama E, Rangaswami A, Krailo M, Maibach R, Rinaldi E, Feng Y, Aronson D, Malogolowkin M et al: The Children's Hepatic tumors International Collaboration (CHIC): Novel 
global rare tumor database yields new prognostic factors in hepatoblastoma and becomes a research model. Eur J Cancer 2016, 52:92-101.

2. Meyers RL, Maibach R, Hiyama E, Haberle B, Krailo M, Rangaswami A, Aronson DC, Malogolowkin $\mathrm{MH}$, Perilongo $\mathrm{G}$, von Schweinitz $\mathrm{D}$ et al: Risk-stratified staging in paediatric hepatoblastoma: a unified analysis from the Children's Hepatic tumors International Collaboration. Lancet Onco/2017, 18(1):122-131.

3. Lim IIP, Bondoc AJ, Geller JI, Tiao GM: Hepatoblastoma-The Evolution of Biology, Surgery, and Transplantation. Children (Basel) 2018, 6(1).

4. Bell D, Ranganathan S, Tao J, Monga SP: Novel Advances in Understanding of Molecular Pathogenesis of Hepatoblastoma: A Wnt/beta-Catenin Perspective. Gene Expr2017, 17(2):141-154.

5. Czauderna P, Garnier H: Hepatoblastoma: current understanding, recent advances, and controversies. F1000Res 2018, 7:53.

6. Hooks KB, Audoux J, Fazli H, Lesjean S, Ernault T, Dugot-Senant N, Leste-Lasserre T, Hagedorn M, Rousseau B, Danet C et al: New insights into diagnosis and therapeutic options for proliferative hepatoblastoma. Hepatology 2018, 68(1):89-102.

7. Adebayo Michael AO, Ko S, Tao J, Moghe A, Yang H, Xu M, Russell JO, Pradhan-Sundd T, Liu S, Singh $S$ et al: Inhibiting Glutamine-Dependent mTORC1 Activation Ameliorates Liver Cancers Driven by beta-Catenin Mutations. Cell Metab 2019, 29(5):1135-1150 e1136.

8. Yang A, Sisson R, Gupta A, Tiao G, Geller Jl: Germline APC mutations in hepatoblastoma. Pediatr Blood Cancer 2018, 65(4).

9. Tao J, Calvisi DF, Ranganathan S, Cigliano A, Zhou L, Singh S, Jiang L, Fan B, Terracciano L, Armeanu-Ebinger $S$ et al: Activation of beta-catenin and Yap1 in human hepatoblastoma and induction of hepatocarcinogenesis in mice. Gastroenterology 2014, 147(3):690-701.

10. Patel SH, Camargo FD, Yimlamai D: Hippo Signaling in the Liver Regulates Organ Size, Cell Fate, and Carcinogenesis. Gastroenterology 2017, 152(3):533-545.

11. Fu V, Plouffe SW, Guan KL: The Hippo pathway in organ development, homeostasis, and regeneration. Curr Opin Cell Biol 2017, 49:99-107.

12. Min Q, Molina L, Li J, Adebayo Michael AO, Russell JO, Preziosi ME, Singh S, Poddar M, Matz-Soja M, Ranganathan $S$ et al: beta-Catenin and Yes-Associated Protein 1 Cooperate in Hepatoblastoma Pathogenesis. Am J Pathol 2019, 189(5):1091-1104.

13. Zhang J, Liu P, Tao J, Wang P, Zhang Y, Song X, Che L, Sumazin P, Ribback S, Kiss A et al: TEA Domain Transcription Factor 4 Is the Major Mediator of Yes-Associated Protein Oncogenic Activity in Mouse and Human Hepatoblastoma. Am J Pathol 2019, 189(5):1077-1090.

14. Callus BA, Finch-Edmondson ML, Fletcher S, Wilton SD: YAPping about and not forgetting TAZ. FEBS Lett 2019, 593(3):253-276.

15. Xin M, Kim Y, Sutherland LB, Qi X, McAnally J, Schwartz RJ, Richardson JA, Bassel-Duby R, Olson EN: Regulation of insulin-like growth factor signaling by Yap governs cardiomyocyte proliferation and embryonic heart size. Sci Signal 2011, 4(196):ra70. 
16. Xu M, Wang J, Xu Z, Li R, Wang P, Shang R, Cigliano A, Ribback S, Solinas A, Pes GM et al: SNAl1 Promotes the Cholangiocellular Phenotype, but not Epithelial-Mesenchymal Transition, in a Murine Hepatocellular Carcinoma Model. Cancer Res 2019, 79(21):5563-5574.

17. Zhang N, Bai H, David KK, Dong J, Zheng Y, Cai J, Giovannini M, Liu P, Anders RA, Pan D: The Merlin/NF2 tumor suppressor functions through the YAP oncoprotein to regulate tissue homeostasis in mammals. Dev Cell 2010, 19(1):27-38.

18. Chen X, Calvisi DF: Hydrodynamic transfection for generation of novel mouse models for liver cancer research. Am J Pathol 2014, 184(4):912-923.

19. Bonamassa B, Hai L, Liu D: Hydrodynamic gene delivery and its applications in pharmaceutical research. Pharm Res 2011, 28(4):694-701.

20. VS T, NA Kk, VF K, VS P, SI S, SA K, NV Eg: Hepatoblastoma, Mouse, in Digestive System. Berlin, Heidelberg: Springer Berlin Heidelberg:; 1997.

21. Calvisi DF, Ladu S, Conner EA, Factor VM, Thorgeirsson SS: Disregulation of E-cadherin in transgenic mouse models of liver cancer. Lab Invest 2004, 84(9):1137-1147.

22. Yimlamai D, Christodoulou C, Galli GG, Yanger K, Pepe-Mooney B, Gurung B, Shrestha K, Cahan P, Stanger BZ, Camargo FD: Hippo pathway activity influences liver cell fate. Cell 2014, 157(6):13241338.

23. Lei QY, Zhang H, Zhao B, Zha ZY, Bai F, Pei XH, Zhao S, Xiong Y, Guan KL: TAZ promotes cell proliferation and epithelial-mesenchymal transition and is inhibited by the hippo pathway. Mol Cell Biol 2008, 28(7):2426-2436.

24. Stauffer JK, Scarzello AJ, Andersen JB, De Kluyver RL, Back TC, Weiss JM, Thorgeirsson SS, Wiltrout $\mathrm{RH}$ : Coactivation of AKT and beta-catenin in mice rapidly induces formation of lipogenic liver tumors. Cancer Res 2011, 71(7):2718-2727.

25. Tward AD, Jones KD, Yant S, Cheung ST, Fan ST, Chen X, Kay MA, Wang R, Bishop JM: Distinct pathways of genomic progression to benign and malignant tumors of the liver. Proc Natl Acad Sci U $S$ A 2007, 104(37):14771-14776.

26. Lee SA, Ho C, Roy R, Kosinski C, Patil MA, Tward AD, Fridlyand J, Chen X: Integration of genomic analysis and in vivo transfection to identify sprouty 2 as a candidate tumor suppressor in liver cancer. Hepatology 2008, 47(4):1200-1210.

27. Soini T, Haveri H, Elo JM, Kauppinen M, Kyronlahti A, Salo MK, Lohi J, Andersson LC, Wilson DB, Heikinheimo M: Transcription factor GATA-4 is abundantly expressed in childhood but not in adult liver tumors. J Pediatr Gastroenterol Nutr 2012, 54(1):101-108.

28. Sumazin P, Chen Y, Trevino LR, Sarabia SF, Hampton OA, Patel K, Mistretta TA, Zorman B, Thompson P, Heczey A et al: Genomic analysis of hepatoblastoma identifies distinct molecular and prognostic subgroups. Hepatology 2017, 65(1):104-121.

29. Zhang H, Liu CY, Zha ZY, Zhao B, Yao J, Zhao S, Xiong Y, Lei QY, Guan KL: TEAD transcription factors mediate the function of TAZ in cell growth and epithelial-mesenchymal transition. J Biol Chem 2009, 284(20):13355-13362. 
30. Raghunath A, Sundarraj K, Arfuso F, Sethi G, Perumal E: Dysregulation of Nrf2 in Hepatocellular Carcinoma: Role in Cancer Progression and Chemoresistance. Cancers (Basel) 2018, 10(12).

31. Menegon S, Columbano A, Giordano S: The Dual Roles of NRF2 in Cancer. Trends Mol Med 2016, 22(7):578-593.

32. Jin X, Moskophidis D, Mivechi NF: Heat shock transcription factor 1 is a key determinant of HCC development by regulating hepatic steatosis and metabolic syndrome. Cell Metab 2011, 14(1):91103.

33. Cigliano A, Pilo MG, Li L, Latte G, Szydlowska M, Simile MM, Paliogiannis P, Che L, Pes GM, Palmieri $G$ et al: Deregulated c-Myc requires a functional HSF1 for experimental and human hepatocarcinogenesis. Oncotarget 2017, 8(53):90638-90650.

34. Cigliano A, Wang C, Pilo MG, Szydlowska M, Brozzetti S, Latte G, Pes GM, Pascale RM, Seddaiu MA, Vidili $G$ et al: Inhibition of HSF1 suppresses the growth of hepatocarcinoma cell lines in vitro and AKT-driven hepatocarcinogenesis in mice. Oncotarget 2017, 8(33):54149-54159.

35. Tschaharganeh DF, Chen X, Latzko P, Malz M, Gaida MM, Felix K, Ladu S, Singer S, Pinna F, Gretz N et al: Yes-associated protein up-regulates Jagged-1 and activates the Notch pathway in human hepatocellular carcinoma. Gastroenterology 2013, 144(7):1530-1542 e1512.

36. Wang J, Dong M, Xu Z, Song X, Zhang S, Qiao Y, Che L, Gordan J, Hu K, Liu Y et al: Notch2 controls hepatocyte-derived cholangiocarcinoma formation in mice. Oncogene 2018, 37(24):3229-3242.

37. Zhang S, Wang J, Wang H, Fan L, Fan B, Zeng B, Tao J, Li X, Che L, Cigliano A et al: Hippo Cascade Controls Lineage Commitment of Liver Tumors in Mice and Humans. Am J Pathol 2018, 188(4):9951006.

38. Litten JB, Chen TT, Schultz R, Herman K, Comstock J, Schiffman J, Tomlinson GE, Rakheja D: Activated NOTCH2 is overexpressed in hepatoblastomas: an immunohistochemical study. Pediatr Dev Pathol 2011, 14(5):378-383.

39. Che L, Fan B, Pilo MG, Xu Z, Liu Y, Cigliano A, Cossu A, Palmieri G, Pascale RM, Porcu A et al: Jagged 1 is a major Notch ligand along cholangiocarcinoma development in mice and humans. Oncogenesis 2016, 5(12):e274.

40. Ma L, Wang X, Jia T, Wei W, Chua MS, So S: Tankyrase inhibitors attenuate WNT/beta-catenin signaling and inhibit growth of hepatocellular carcinoma cells. Oncotarget 2015, 6(28):25390-25401.

41. Jia J, Qiao Y, Pilo MG, Cigliano A, Liu X, Shao Z, Calvisi DF, Chen X: Tankyrase inhibitors suppress hepatocellular carcinoma cell growth via modulating the Hippo cascade. PLOS One 2017, 12(9):e0184068.

\section{Tables}

Table 1. Clinicopathological features of human hepatoblastoma patients 


\begin{tabular}{lc}
\hline Variables & \\
\hline No. of patients & 32 \\
Male & 18 \\
Female & 14 \\
Age (years) & $4.0 \pm 2.6$ \\
& \\
Tumor morphology & \\
Fetal & 14 \\
Embryonal & 8 \\
Mixed & 8 \\
Small undifferentiated & 2 \\
& \\
Recurrence & \\
Yes & 12 \\
No & 20 \\
Lung metastases & \\
Yes & 10 \\
No & 22 \\
\hline
\end{tabular}

Table 2. Primary antibodies used for immunohistochemistry (IHC) and Western blot analysis (WB) 


\begin{tabular}{|c|c|c|c|c|}
\hline Antibody name & Use & Concentration & Company & Catalogue No \\
\hline CK19 & IHC & $1: 400$ & Abcam & Ab133496 \\
\hline CK7 & $\mathrm{IHC}$ & $1: 400$ & Abcam & Ab181598 \\
\hline $\operatorname{Hnf}-4 \alpha$ & IHC & $1: 100$ & Abcam & Ab181604 \\
\hline $\mathrm{Ki}-67$ & IHC & $1: 100$ & Bethyl & IHC-00375 \\
\hline E-cadherin & IHC & $1: 100$ & Cell Signaling Technology & 3195 \\
\hline Myc-tag & $\mathrm{IHC} ; \mathrm{WB}$ & $1: 100 ; 1: 1000$ & MMCRI & Vli01 \\
\hline Vimentin & IHC & 1:1000 & Abcam & Ab92547 \\
\hline Desmin & IHC & $1: 200$ & Abcam & $\mathrm{Ab} 15200$ \\
\hline V5-tag & IHC & $1: 100$ & Abcam & Ab95038 \\
\hline Yap & $\mathrm{IHC} ; \mathrm{WB}$ & $1: 100 ; 1: 1000$ & Cell Signaling Technology & 14074 \\
\hline$\alpha-\mathrm{SMA}$ & IHC & $1: 400$ & Abcam & Ab5994 \\
\hline TAZ/WWTR1 & IHC & $1: 100$ & Sigma-Aldrich & AMab90730 \\
\hline TAZ & IHC & $1: 100$ & BD Biosciences & 560235 \\
\hline Yap/Taz & WB & 1:1000 & Cell Signaling Technology & 8418 \\
\hline Notch2 & IHC & $1: 50$ & Cell Signaling Technology & 5732 \\
\hline FOXA1 & IHC & $1: 400$ & Abcam & Ab170933 \\
\hline FOXA2 & IHC & $1: 200$ & Abcam & Ab108422 \\
\hline CEBPA & IHC & $1: 100$ & Cell Signaling Technology & 8178 \\
\hline GLUL & IHC & $1: 500$ & BD Biosciences & 610517 \\
\hline CD44v6 & IHC & $1: 100$ & ThermoFischer Scientific & MA1-81995 \\
\hline Nanog & IHC & $1: 100$ & Abcam & Ab214549 \\
\hline CD10 & IHC & $1: 100$ & Abcam & Ab256494 \\
\hline GATA4 & IHC & $1: 100$ & Abcam & Ab84593 \\
\hline EPCAM & IHC & $1: 100$ & Abcam & Ab221552 \\
\hline ERG & IHC & $1: 100$ & Abcam & Ab92513 \\
\hline CD34 & IHC & $1: 150$ & Abcam & Ab8158 \\
\hline
\end{tabular}




\begin{tabular}{|c|c|c|c|c|} 
NCAM1/CD56 & IHC & $1: 400$ & Cell Signaling Technology & 99746 \\
\hline HSF1 & IHC & $1: 100$ & Cell Signaling Technology & 12972 \\
\hline$\beta$-Catenin & IHC; WB & $1: 100 ; 1: 1000$ & BD Biosciences & 610153 \\
\hline GAPDH & WB & $1: 10000$ & EMD Millipore & AB2302 \\
\hline$\beta$-Actin & WB & $1: 5000$ & Santa Cruz Biotechnology & sc-10731 \\
\hline
\end{tabular}

Table'3. Validated Gene Expression Assays used for real-time qRT-PCR experiments 


\begin{tabular}{|c|c|}
\hline Name & Catalog number \\
\hline Mouse Afp & Mm00431715_m1 \\
\hline Mouse GPC3 & Mm00516722_m1 \\
\hline Mouse Dlk1 & Mm00494477_m1 \\
\hline Mouse Cited1 & Mm01235642_g1 \\
\hline Mouse Epcam & Mm00493214_m1 \\
\hline Mouse Gata4 & Mm00484689_m1 \\
\hline Mouse Nanog & Mm02019550_s1 \\
\hline Mouse Vim & Mm01333430_m1 \\
\hline Mouse Des & Mm00802455_m1 \\
\hline Mouse Axin 2 & Mm00443610_m1 \\
\hline Mouse Glul & Mm00725701_s1 \\
\hline Mouse Tbx3 & Mm01195726_m1 \\
\hline Mouse Ctgf & Mm01192933_g1 \\
\hline Mouse Cyr61 & Mm00487498_m1 \\
\hline Mouse Jag 1 & Mm00496902_m1 \\
\hline Mouse Notch1 & Mm00627185_m1 \\
\hline Mouse Notch2 & Mm00803077_m1 \\
\hline Mouse Hes1 & Mm01342805_m1 \\
\hline Mouse Nrf2 & Mm00477784_m1 \\
\hline Mouse Keap1 & Mm00497268_m1 \\
\hline Mouse Nqo1 & Mm01253561_m1 \\
\hline
\end{tabular}




\begin{tabular}{|c|l|} 
Mouse Hsf1 & Mm01201402_m1 \\
\hline Mouse $\beta$-Actin & Mm00607939_s1 \\
\hline Human TAZ & Hs00210007_m1 \\
\hline Human YAP & Hs00902712_g1 \\
\hline Human HSF1 & Hs00232134_m1 \\
\hline Human $\beta$-Actin & Hs01060665_g1 \\
& \\
\hline
\end{tabular}

\section{Figures}

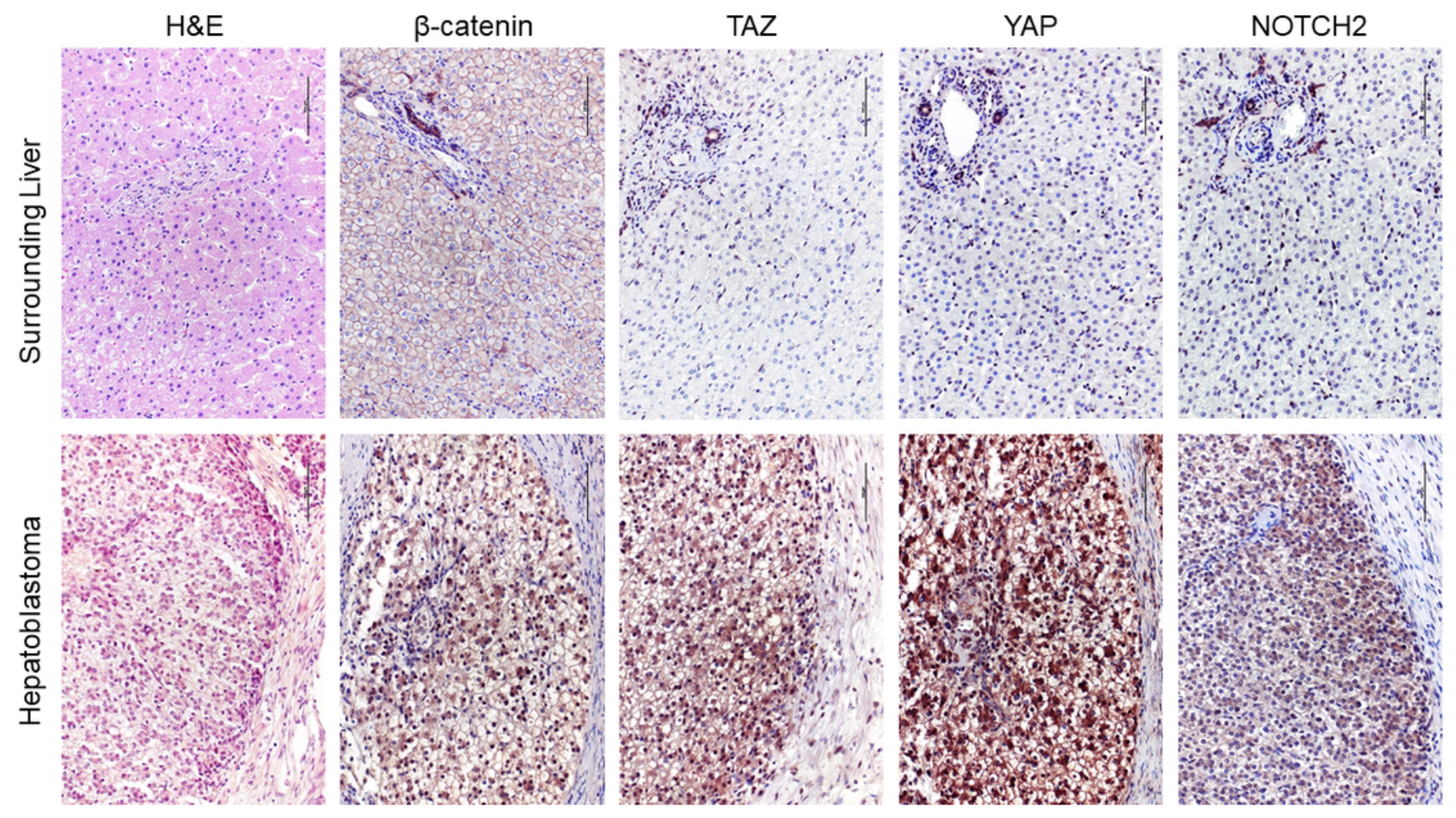

Figure 1 
Representative immunohistochemical patterns of $\beta$-catenin, TAZ, YAP, and NOTCH2 proteins in human normal liver and hepatoblastoma. Upper panel: normal liver, lower panel: hepatoblastoma (fetal subtype). Magnification: 200x; scale bar $=100 \mu \mathrm{m}$.

A

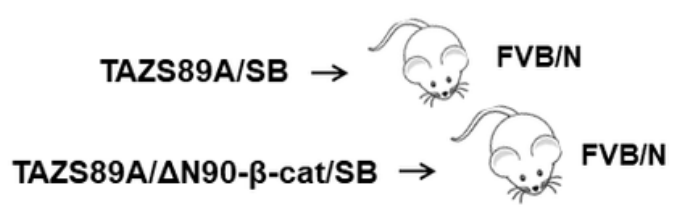

C

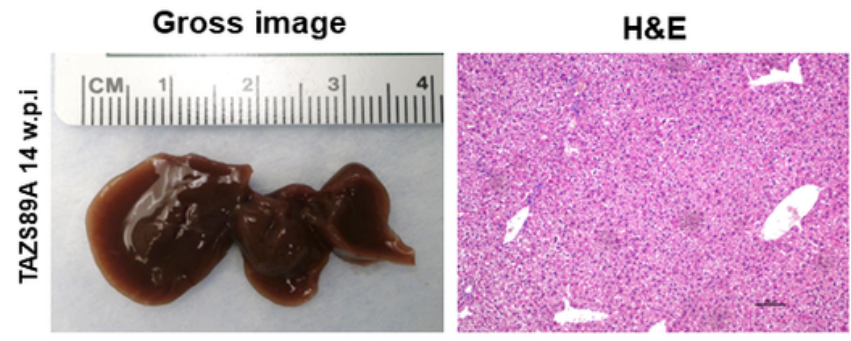

D

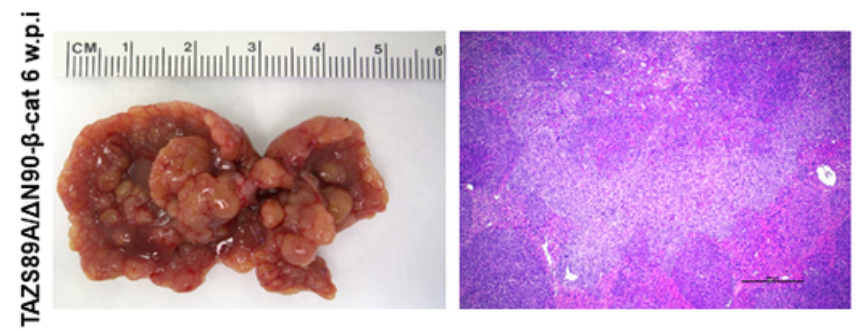

B

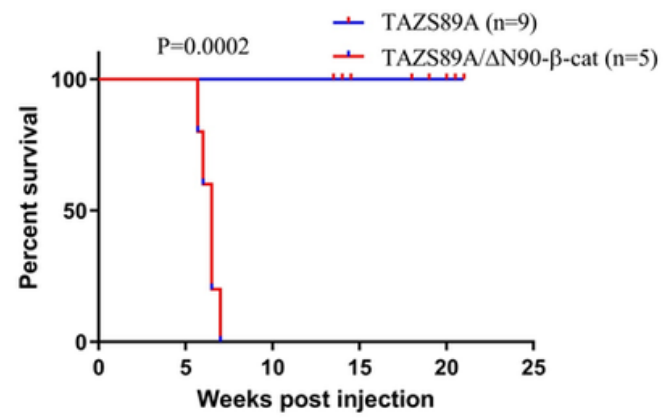

E

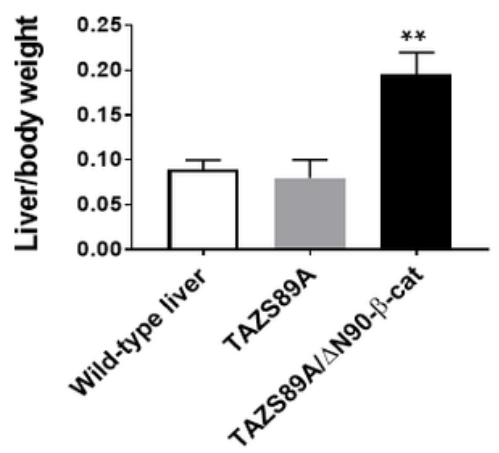

\section{Figure 2}

Overexpression of a constitutively activated TAZ (TAZS89A) form is unable to trigger tumor development alone but cooperates with oncogenic $\beta$-Catenin to induce HB development in mice. (A) Scheme of the experiments conducted. (B) Survival curves of TAZS89A and TAZS89A/ $\triangle N 90-\beta$-catenin mice. (C,D) Gross and microscopic images of TAZS89A and TAZS89A/ $\triangle N 90-\beta$-catenin mouse livers. Note the normal histologic features of the TAZS89A-only injected liver (C), whereas almost the whole parenchyma of TAZS89A/ $\triangle$ N90- $\beta$-catenin mice is occupied by tumor lesions (D). (E) Liver weight/body weight ratio of normal (wild-type) livers as well as TAZS89A and TAZS89A/ $\triangle N 90-\beta$-catenin mice $(n=5$ for each mouse group examined). Statistical significance: $* * P<0.01$ vs wild-type and TAZS8A mice. Original magnification: 100x in C and 40x in D; scale bar: $100 \mu \mathrm{m}$ in $\mathrm{C}$ and $500 \mu \mathrm{m}$ in D. Abbreviations: H\&E, hematoxylin and eosin staining; SB, sleeping beauty transposase; w.p.i, weeks post injection. 

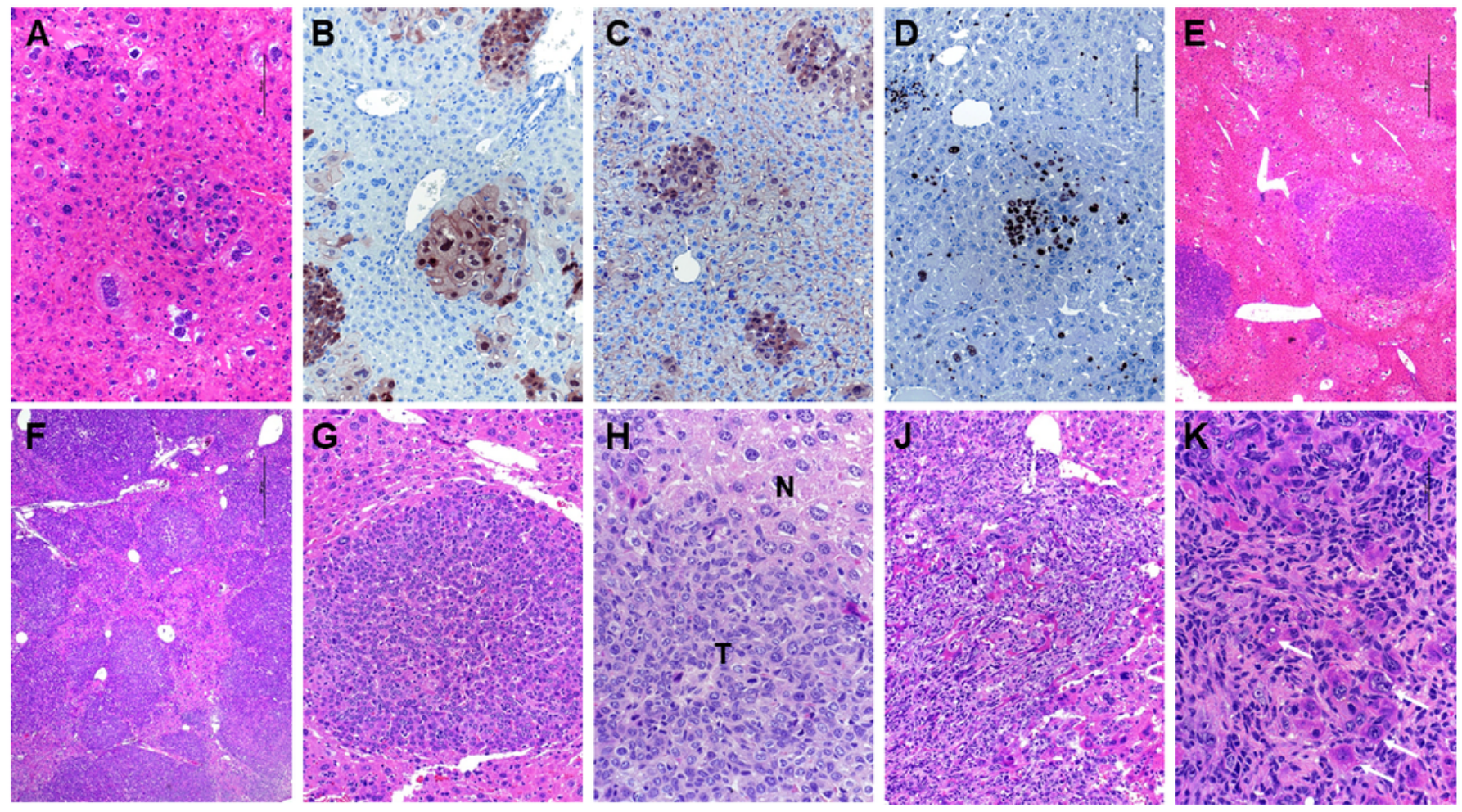

\section{Figure 3}

Histopathological characterization of liver lesions developed in TAZS89A/ $\triangle N 90-\beta$-catenin mice. (A) Hematoxylin and eosin (H\&E) staining of a TAZS89A/ $\triangle$ N90- $\beta$-catenin mice mouse liver 1.5 weeks after hydrodynamic tail vein injection. Altered cells characterized mainly by nuclei of various size form little clusters in the liver parenchyma. These cells express the Myc-tagged $\beta$-catenin (B) and TAZ (C) proteins, indicating their origin from injected constructs, and are actively proliferating ( $D$; as indicated by nuclear immunoreactivity for the proliferation marker Ki67). (E) By 3 weeks post injection, nodules of altered cells are appreciable throughout the liver. These nodular lesions occupy most of the liver surface by 6 weeks post injection (E). (F) Lesions appear composed of small cells with prominent nuclei and a high nuclear/cytoplasmic ratio, resembling human fetal and crowded fetal hepatoblastomas. (G) At higher magnification, the small size of tumor cells $(T)$ when compared to normal hepatocytes $(N)$ can be appreciated. $(\mathrm{J})$ Intriguingly, together with the epithelial component shown in (F), many tumors displayed the presence of a mesenchymal part, characterized by the presence of spindle cells and a stroma component. (K) Within the spindle and stromal cells, epithelial/hepatoblast-like cells can be appreciated (indicated by arrows). Original magnifications: 200x in A,B,C,D,G,J; 40x in E and F; 400x in $\mathrm{H}$ and $\mathrm{K}$. 

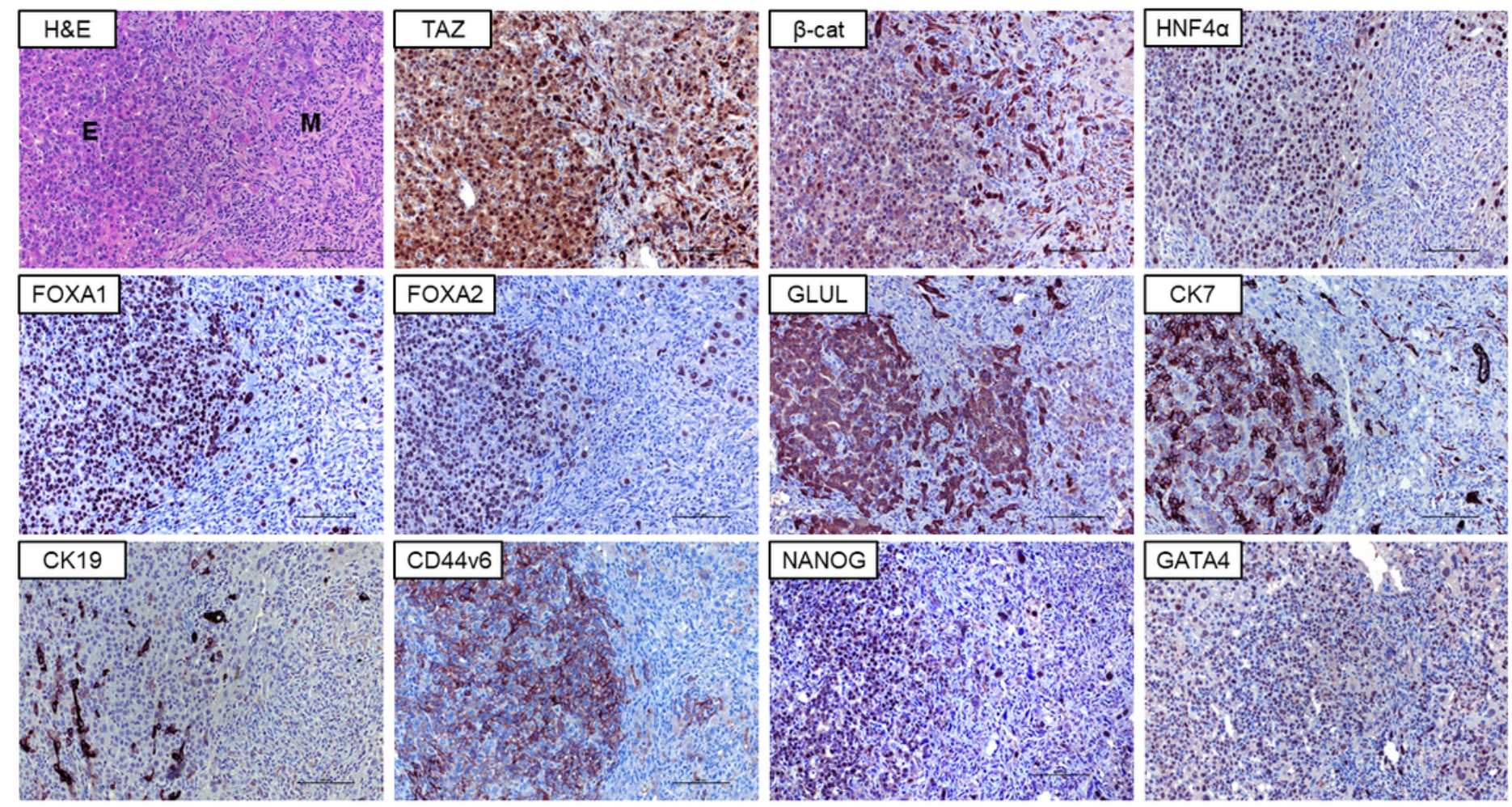

\section{Figure 4}

Molecular characterization of liver lesions developed in TAZS89A/ $\triangle$ N90- $\beta$-catenin mice by IHC. Staining patterns of the epithelial $(E)$ and the mesenchymal $(M)$ component are shown in each picture. Original magnification: 200x. 
A

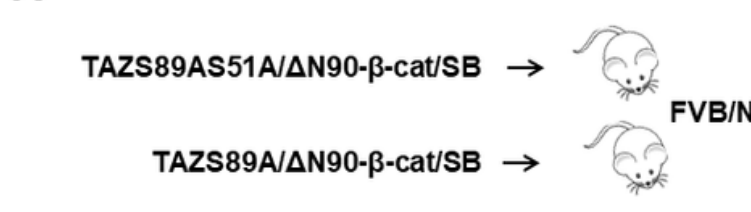

C

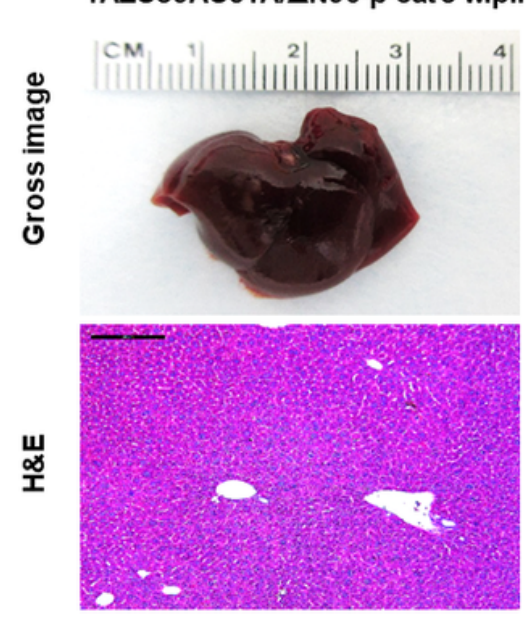

B

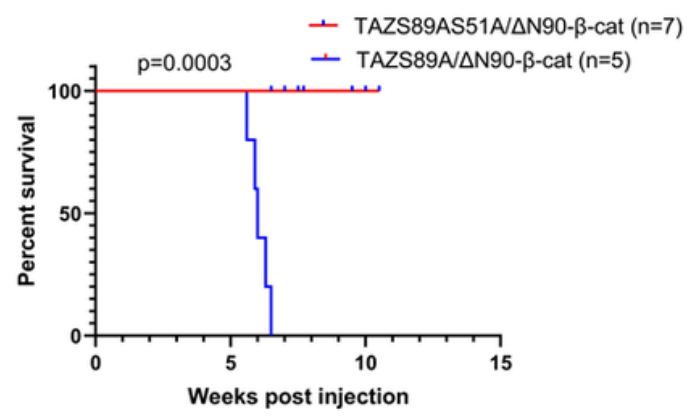

D

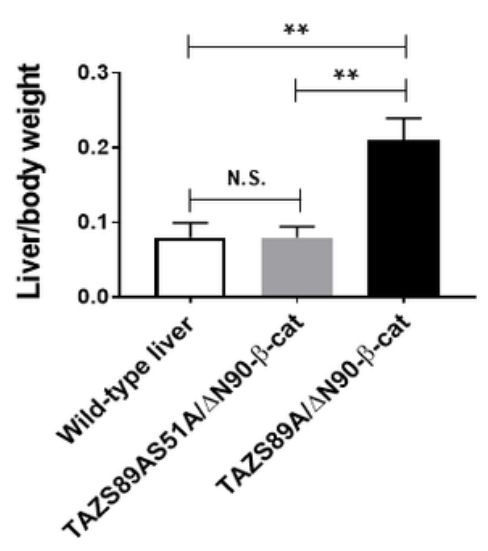

\section{Figure 5}

Transcriptionally active TAZ is required for hepatocarcinogenesis in association with oncogenic $\beta$ catenin. (A) Scheme of the experiment. (B) Survival curve of TAZS89A/ $\triangle N 90-\beta$-catenin and TAZS89AS51A/ $\Delta$ N90- $\beta$-catenin mice. TAZS89AS51A overexpression results in the induction of TAZdependent effects that are independent of its binding to TEAD transcription factors. (C) Impairment of TAZ-TEAD binding by TAZS89AS51A inhibits TAZS89A/ $\beta$-catenin carcinogenesis in the mouse liver. (D) Liver weight/body weight ratio of wild-type as well as TAZS89A/ $\triangle$ N90- $\beta$-catenin and TAZS89AS51A/ $\Delta$ N90- $\beta$-catenin mice. Original magnification: $200 x$ in $C$. Abbreviations: $\mathrm{H} \& \mathrm{E}$, hematoxylin and eosin staining; SB, sleeping beauty transposase. 
A

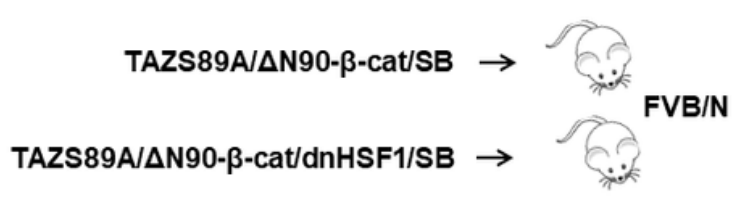

C

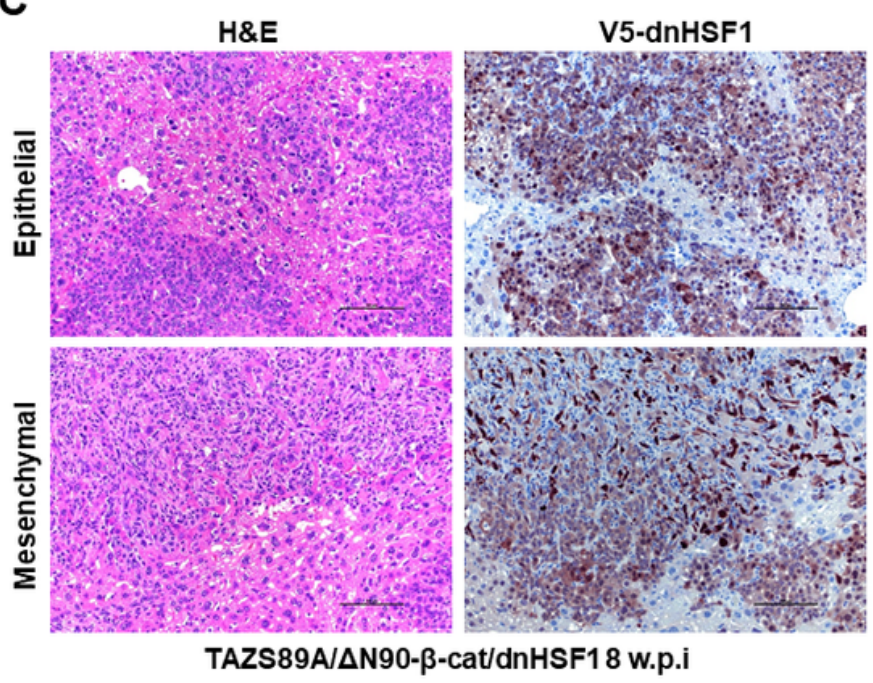

B

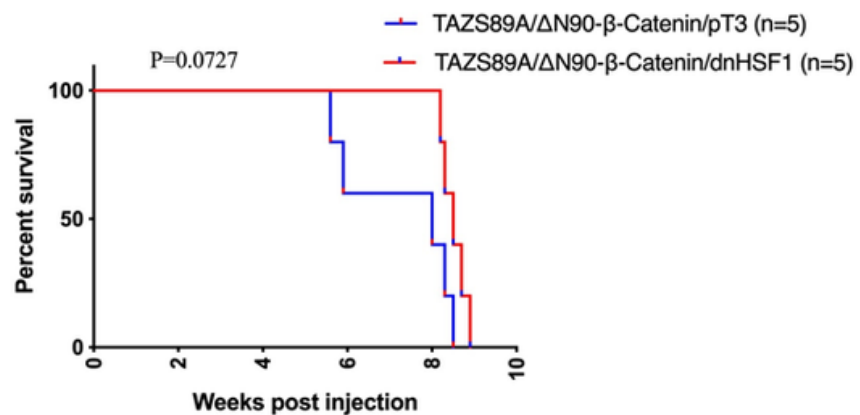

\section{Figure 6}

Inactivation of HSF1 does not impair liver carcinogenesis in TAZS89A/ $\triangle N 90-\beta$-catenin mice. (A) Study design. (B) Survival curve of TAZS89A/ $\Delta N 90-\beta$-catenin and TAZS89A/ $\triangle N 90-\beta$-catenin/dnHSF1 mice. The dnHSF1 construct is a dominant negative version of HSF1 that suppresses HSF1 activity. (C) Absence of any effect on tumor development in TAZS89A/ $\triangle$ N90- $\beta$-catenin/dnHSF1 mice. Tumors from TAZS89A/ $\triangle$ N90- $\beta$-catenin/dnHSF1 mice display the presence of the epithelial and mesenchymal component. Homogeneous immunohistochemical staining for the V5-tagged dnHSF1 construct throughout the lesions indicates the effective transfection of dnHSF1 in the tumor cells. (D) Liver weight/body weight ratio of wild-type as well as TAZS89A/ $\triangle N$ N90- $\beta$-catenin and TAZS89A/ $\Delta N 90-\beta-$ catenin/dnHSF1 mice. Original magnification: 200x in C. Abbreviations: H\&E, hematoxylin and eosin staining; SB, sleeping beauty transposase. 
A
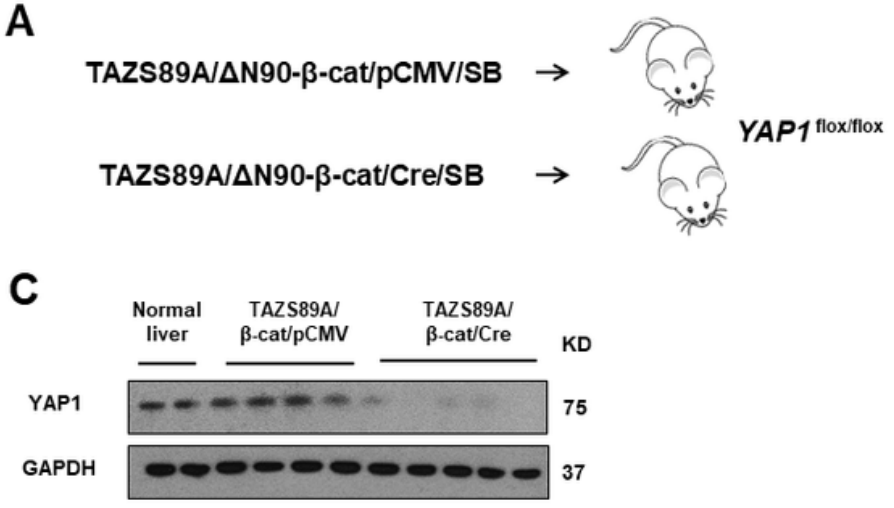

D

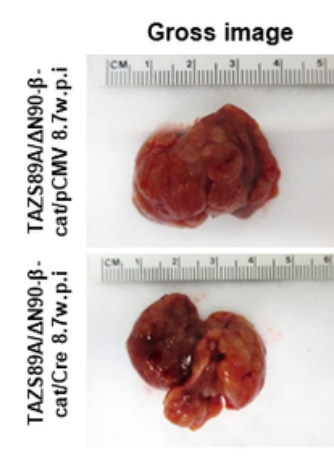

B

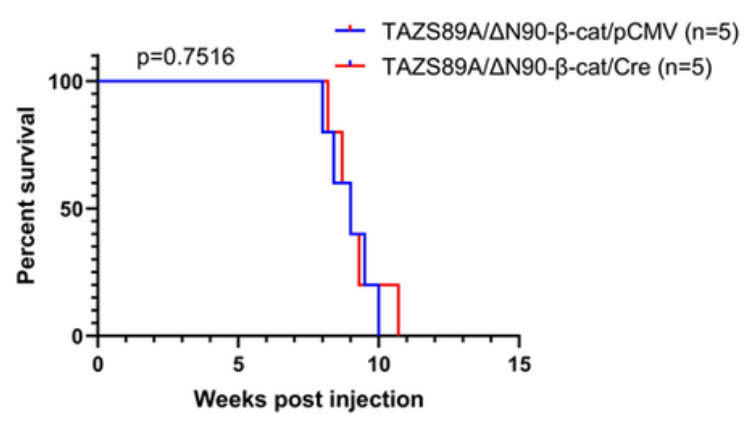

E
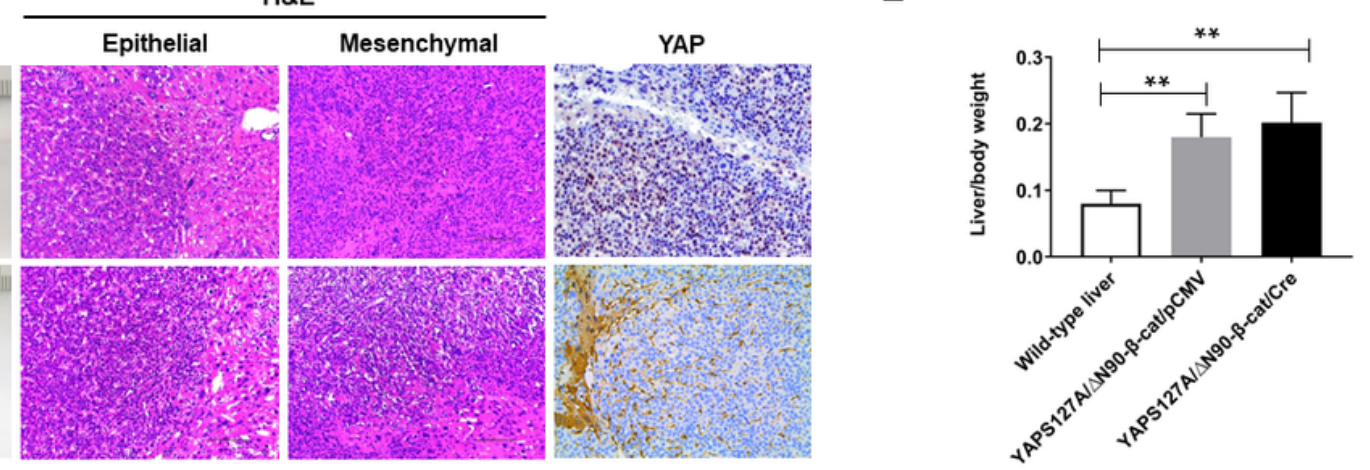

Figure 7

Deletion of Yap does not affect liver carcinogenesis in TAZS89A/ $\Delta$ N90- $\beta$-catenin mice. (A) Study design. Briefly, Yapflox/flox conditional knockout mice were subjected to hydrodynamic tail vein injection of either TAZS89A/ $\triangle$ N90- $\beta$-catenin/pCMV (control) or TAZS89A/ $\Delta N 90-\beta$-catenin/Cre plasmid. (B) Survival curve of TAZS89A/ $\triangle$ N90- $\beta$-catenin/pCMV and TAZS89A/ $\triangle N$ N0- $\beta$-catenin/Cre mice. (C) Western blot analysis showing the effective Cre-mediated deletion of Yap in the liver of TAZS89A/ $\triangle N 90-\beta$-catenin/Cre mice. (D) Liver lesions from TAZS89A/ $\Delta$ N90- $\beta$-catenin/pCMV or TAZS89A/ $\Delta N 90-\beta$-catenin/Cre mice are morphologically undistinguishable, both consisting of an epithelial and a mesenchymal component. As expected, TAZS89A/ $\triangle$ N90- $\beta$-catenin/pCMV livers display nuclear accumulation of Yap in the lesions (upper panel), which is instead lost in corresponding lesions from TAZS89A/ $\Delta$ N90- $\beta$-catenin/Cre mice. In the latter mice, Yap cytoplasmic immunoreactivity is preserved in the non-neoplastic liver surrounding the tumors (lower panel). (D) Liver weight/body weight ratio of wild-type as well as TAZS89A/ $\triangle N$ N00- $\beta$ catenin/pCMV and TAZS89A/ $\triangle$ N90- $\beta$-catenin/Cre mice. Original magnification: 200x in C. Abbreviations: $H \& E$, hematoxylin and eosin staining; $S B$, sleeping beauty transposase. 
A

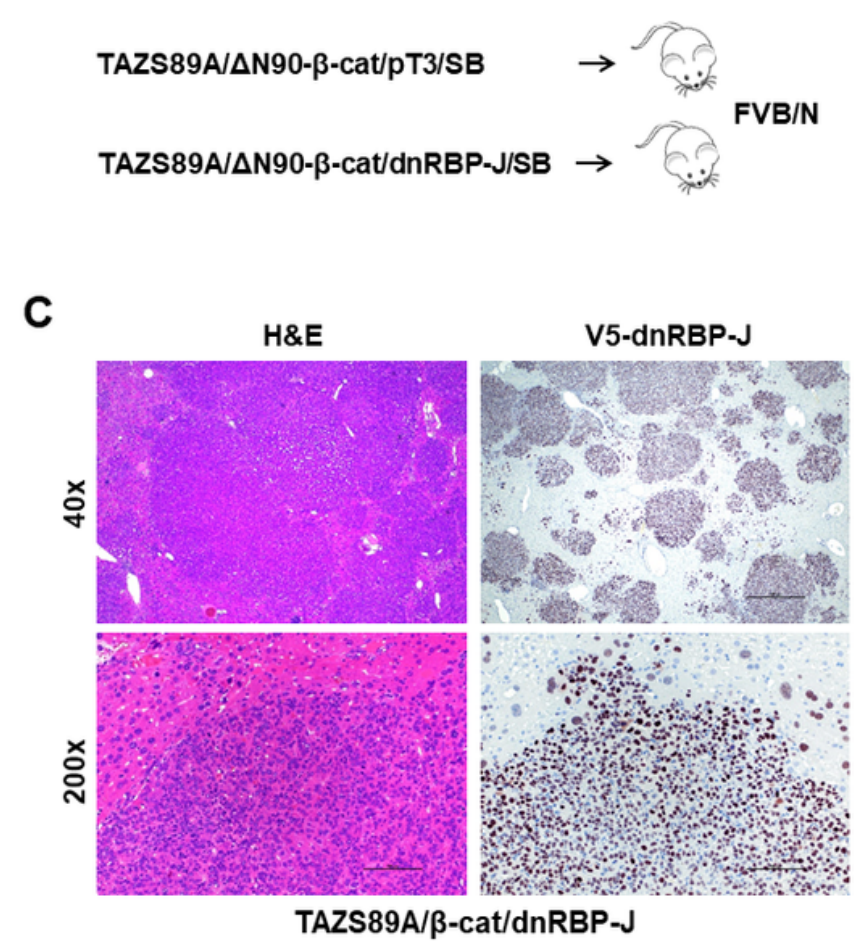

B

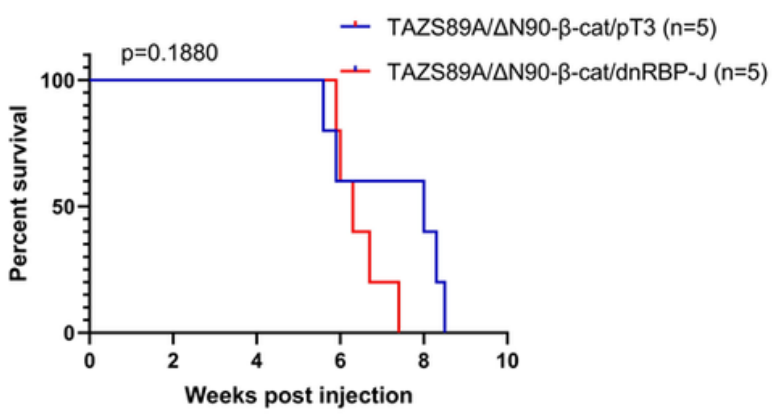

D

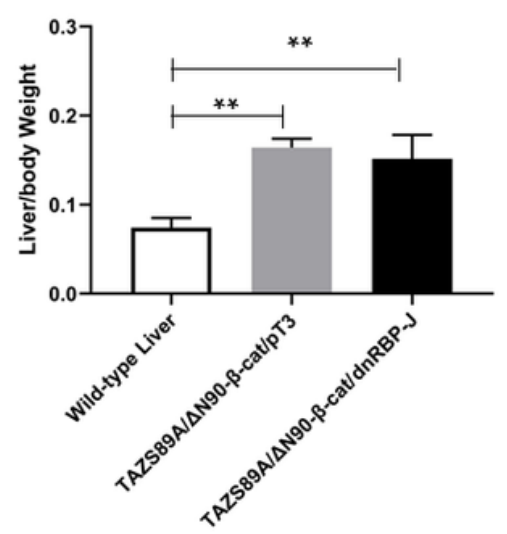

\section{Figure 8}

Inactivation of the Notch cascade abolish the mesenchymal component of liver tumors, without impairing carcinogenesis, in TAZS89A/ $\triangle N$ N0- $\beta$-catenin mice. (A) Study design. Briefly, wild-type mice were subjected to hydrodynamic tail vein injection of either TAZS89A/ $\Delta$ N90- $\beta$-catenin/pT3 (control) or TAZS89A/ $\triangle$ N90- $\beta$-catenin/dnRBP-J plasmid. dnRBP-J is the dominant negative form of the transcriptional coactivator RBP-J, whose overexpression effectively inhibits the canonical Notch cascade.

(B) Survival curve of TAZS89A/ $\Delta$ N90- $\beta$-catenin/pT3 (control) or TAZS89A/ $\Delta N 90-\beta$-catenin/dnRBP-J mice. (C) Liver lesions from TAZS89A/ $\triangle N$ 90- $\beta$-catenin/dnRBP-J mice consist only of an epithelial component. Homogeneous immunohistochemical staining for the V5-tagged dnRBP-J construct throughout the lesions indicates the effective transfection of the dnRBP-J plasmid in the tumor cells. (D) Liver weight/body weight ratio of wild-type as well as TAZS89A/ $\triangle N$ N0- $\beta$-catenin/pT3 (control) or TAZS89A/ $\Delta$ N90- $\beta$-catenin/dnRBP-J mice. Original magnification: 40x (upper panel) and 200x (lower panel) in C. Abbreviations: $\mathrm{H} \& \mathrm{E}$, hematoxylin and eosin staining; SB, sleeping beauty transposase. 